\title{
On channel assignment and multicast routing in multi-channel multi-radio wireless mesh networks
}

\author{
Mohsen Jahanshahi* \\ Computer Engineering Department, \\ Sciences \& Research Branch, Islamic Azad University, \\ P.O. Box: 1477893855 , \\ Hesarak, Tehran, I.R. Iran, \\ E-mail: mjahanshahi@iauctb.ac.ir \\ ${ }^{*}$ Corresponding author
}

\section{Mehdi Dehghan and Mohammad Reza Meybodi}

Computer Engineering Department,

Amirkabir University of Technology,

No. 424, Hafez Ave, P.O. Box: 15875-4413,

Tehran, Iran

E-mail: dehghan@aut.ac.ir

E-mail:mmeybodi@aut.ac.ir

\begin{abstract}
Multicast is a key networking service, enabling one-shot delivery of information from a source to multiple destinations and is considered underlying for collaborating multimedia applications such as video conferencing, distance learning and other forms of content distributing over Multi-Channel Multi-Radio Wireless Mesh Networks (MCMR WMNs). Multicast protocol as designed in these networks, however, is tightly coupled with the specifics of the nodes' channel-radio associations to realise minimum interference communication. The mainstream of research in WMN multicasting is oriented towards heuristic or meta-heuristic strategies which basically take on a sequential approach to solve the channel assignment and the multicast routing as two disjoint sub-problems. The resulting network configurations would be sub-optimal in this case. It is given that the cross-interaction between the two sub-problems is an effect of the problem's specifications. In this paper, first, we propose a cross-layer mathematical formulation of joint channel assignment and multicast tree construction in MCMR WMNs, which, opposed to the existing schemes guarantees optimal solution. The simulation results demonstrate that our cross-layer design outperforms the Level Channel Assignment (LCA), Multi-Channel Multicast (MCM), the Genetic Algorithm (GA), Simulated Annealing (SA) and the Tabu Search (TS)-based methods proposed by Zeng et al. (2010) and Cheng et al. (2011) respectively, in terms of inter-channel interference. Second, since joint optimisation modelling has been relatively demanding in terms of complexity, we relax the optimality requirement and alternatively explore the option of a layered formulation in which to ensure an optimal solution for each sub-problem. Our alternative design is proved superior to the prior art in terms of interference minimisation too. We conduct an extensive series of simulations to analyse the optimality and complexity of our two design strategies. The overall result of the interference, is our optimality measurement. Also, complexity is evaluated in terms of the memory consumption as well as the required time to solve the multicast problem.
\end{abstract}

Keyword: WMN; wireless mesh network; multi-channel; multi-radio; channel assignment; multicast tree construction; cross-layer design; BIP; binary integer programming.

Reference to this paper should be made as follows: Jahanshahi, M., Dehghan, M. and. Meybodi, M.R. (2013) 'On channel assignment and multicast routing in multi-channel multiradio wireless mesh networks', Int. J. Ad Hoc and Ubiquitous Computing, Vol. 12, No. 4, pp.225-244.

Biographical notes: Mohsen Jahanshahi completed his BS and MS in Computer Engineering in Iran in 2002 and 2005, respectively. He also achieved a PhD in Computer Engineering from the Islamic Azad University (Tehran Science and Research Branch) in 2011. He joined the faculty of the Computer Engineering Department at Islamic Azad University (Central Tehran Branch) in 2006. His research interests include wireless mesh networks, wireless sensor networks, cognitive networks, mathematical optimisation, learning systems and soft computing. 
Mehdi Dehghan received his BS in Computer Engineering from the Iran University of Science and Technology (IUST), Tehran, Iran, in 1992 and his MS and PhD from Amirkabir University of Technology (AUT), Tehran, Iran, in 1995 and 2001, respectively. He is an Associate Professor of Computer Engineering and Information Technology at Amirkabir University of Technology (AUT). Prior to joining AUT in 2004, he was a Research Scientist at the Iran Telecommunication Research Center (ITRC), working in the area of quality-of-service provisioning and network management. His research interests are in wireless networks, pattern recognition, fault-tolerant computing and distributed systems.

M.R. Meybodi received his BS and MS in Economics from the Shahid Beheshti University in Iran in 1973 and 1977, respectively. He also received his MS and $\mathrm{PhD}$ in Computer Science from Oklahoma University, USA, in 1980 and 1983, respectively. Currently, he is a Full Professor in the Computer Engineering Department of Amirkabir University of Technology, in Tehran, Iran. Prior to his current position, he worked from1983 to 1985 as Assistant Professor at Western Michigan University and from 1985 to 1991 as Associate Professor at Ohio University, USA. His research interests include channel management in cellular networks, learning systems, parallel algorithms, soft computing and software development.

\section{Introduction}

The Wireless Mesh Network (WMN) has been envisioned as the economically viable networking paradigm for scalable QoS-aware delivery of heterogeneous traffic over broadband and large-scale wireless commodity networks (Wang et al., 2005; Martinez and Bafalluy, 2010). WMNs consist of mesh routers and mesh clients, where mesh routers, as opposed to nodes in a mobile/static ad hoc network (MANET) (Jeong et al., 2007; Yang and Wang, 2009; Rabara and Vijayalekshmi, 2011; Palanisamy and Annadurai, 2011; Cao et al., 2009; Mohamed and Alnuweiri, 2009) have minimal mobility and form the backbone of WMNs. They also provide network access for both mesh and conventional clients (Wang et al., 2005). Compared to Wireless Sensor Networks (WSNs) (Ye and Cheng, 2007), mesh nodes are not energy constrained. However, the main objective in these networks is physical layer capacity maximisation through the interference minimisation, which is typically achieved by having each node transceive through multiple radios tuned on multiple channels (Bahl et al., 2004; Ma et al., 2008) thus being a participant in a number of parallel communications (Gupta and Kumar, 2000).

Some of the key applications for Multi-Channel Multi-Radio WMNs (MCMR WMNs) are multicastbased systems such as video conferencing, online games, webcast and distance learning, etc. However, while wireless communication is intrinsically apt for performing multicast routing due to the broadcast nature of the air medium, inter-channel interference in WMNs plays a key factor in determining the actual data rate achievable for a multicast service. Wireless interference occurs when two links within a two-hop distance of each other are assigned to the same channel. The interference caused by channel conflict diminishes the performance of the wireless communication dramatically. Therefore, for multicast routing, each link on the multicast tree should be assigned to a channel such that minimal interference occurs. As clearly stipulated in the
Protocol model (Gupta and Kumar, 2000; Kodialam and Nandagopal, 2005; Hossain and Leung, 2008), which is also the interference model underlying the formulation presented in this paper, minimising the number of interfering links in a wireless mesh network would have a direct impact on throughput maximisation, as also corroborated in Zeng et al. (2007, 2010) and Cheng and Yang (2011).

Clearly, multicast routing in WMNs not only the best routing tree's establishment requires but we also need to assign proper channels to its links. In MCMR WMNs, interference minimisation is normally tackled by devising channel assignment schemes in the MAC layer. Efficient channel-radio associations often come at the expense of satisfying more complex connectivity constraints; in particular, for two nodes to be connected, not only should they be within transmission radius of one another, but also that the same channel should be assigned to their radios. Multicast routing, under this additional constraint, is even more complicated, since the multicast tree now has to be constructed for the channel-radio association, which also minimises inter-channel interference.

Despite its vast number of applications and practical importance, few works have specifically been targeted at multicast performance optimisation in MCMR WMNs. The mainstream of research in this area has considered channel assignment and multicast routing as two disjoint sub-problems to be solved in sequence (Zeng et al., 2007, 2010; Cheng and Yang, 2008a, 2008b, 2011; Lim et al., 2009), as envisaged in Nguyen and Nguyen (2008, 2009a, 2009b) and Yin et al. (2007), it might even be the case that the solution for either one of these two sub-problems is assumed to be previously calculated and given as input to the other. The downside associated with these schemes, however, is that the cross-interaction between the two sub-problems would not be accounted for and that their reliance on heuristic or meta-heuristic initiatives does not come up with the optimal solution.

Networking problems are ideally formulated in mathematical terms so that the resulting configurations 
and performance tuning parameters can be optimised with ultimate guarantee. However, to date, we are unaware of any published study that has mathematically approached the cross-optimisation of channel assignment and multicast tree construction problems for MCMR WMNs.

In this paper, we propose a cross-layer design for joint optimisation of channel assignment and multicast tree construction problems. As opposed to the prior art, the two sub-problems would be solved conjointly and their interplay is thoroughly accounted for. The mathematical formulation adopted in this paper is based on a Binary Integer Programming (BIP) model which, compared to the previous heuristic or meta-heuristic-based models, guarantees an optimal solution. We argue that linear programming-based formulations suit the typically low-density and limited scale of WMN settings (Nguyen and Xu, 2007; Nguyen, 2008). Such formulations also come with the advantage of easy extensibility in the sense that their constraint-based problem definition can simply be tailored to fit a new design context. The application of BIP, especially in our cross-optimisation setting, yields a channel assignment scheme which fully exhausts the pool of available resources (i.e., channels and radios) so as to construct the optimum (viz. minimal interference) multicast routing structure. Our modelling essentially provides a frame of reference to evaluate comparable centralised or distributed schemes by giving the optimality of the outcome of a BIP formulation.

Simulation results reveal that our proposed crosslayer design outperforms the two heuristic-based schemes discussed in Zeng et al. (2007, 2010), namely Level Channel Assignment (LCA) and Multi-Channel Multicast (MCM), as well as the methods based on the Genetic Algorithm (GA), Simulated Annealing (SA) and the Tabu Search (TS) proposed in Cheng and Yang (2008a, 2008b, 2011), in terms of interference suffering by the network. Furthermore, since joint optimisation modelling is relatively demanding in terms of complexity, we relax the optimality requirement and alternatively explore the option of a layered formulation to ensure an optimal solution for each sub-problem instead. As evidenced by the outcome of the simulations, the interference associated with the layered approach, though marginally higher than that of the cross-layer design, is still significantly smaller compared to those of LCAs, MCMs and methods based on GA, SA and TS. The efficiency of our framework is further investigated by contrasting the characteristics of our layered and cross-layer designs in terms of both optimality and complexity. The metrics used to measure the optimality of the two proposed approaches includes the interference suffered by the network. On the other hand, complexity is measured in terms of memory consumption as well as the time required to solve the multicast problem. In addition, the time complexity of two proposed methods is analysed.

We also explicitly address the hidden channel problem (Lim et al., 2009) which occurs when two nodes within a twohop distance, which are tuned on the same channel, attempt to send or receive data simultaneously.

The reminder of this paper is organised as follows: In Section 2, we briefly review the existing multicast routing methods in MCMR WMNs, highlighting their strengths and shortcomings. Details of the mathematical formulations for the proposed layered and cross-layered schemes are presented in Section 3. In Section 4, the issues of connectivity and loop occurrence are investigated for our framework. Section 5 is dedicated to a comparative simulation study together with the analysis of the optimality and complexity of our solutions. In Section 6, two proposed designs are further investigated with respect to time complexity and memory demands. We conclude the paper in Section 7.

\section{Related works}

As also pointed out in the Introduction, to achieve high performance in WMNs, interference should be reduced. One of the most significant interference reduction techniques is channel assignment, which specifies the channel to be assigned to a radio in such a way that there is the least contribution to the overall interference. Channel assignment for unicast routing in MCMR WMNs has been addressed extensively in the literature (e.g., Kodialam and Nandagopal (2005), Skalli et al. (2007), Subramanian et al. (2008), Rad and Wong (2006), Ramachandran et al. (2006), Marina et al. (2010), Das et al. (2005), Alicherry et al. (2005), Tang et al. (2005), Raniwala et al. (2004) and Tasaki et al. (2004). The mainstream of research in this area can be classified in two categories. The first is disjoint, in which channel assignment is performed on a given routing topology ( $\mathrm{Rad}$ and Wong, 2006; Das et al., 2005; Raniwala et al., 2004), or routing is accomplished over a given channel assignment scheme (Subramanian et al., 2008; Ramachandran et al., 2006; Marina et al., 2010; Tang et al., 2005). The second is joint methods (Kodialam and Nandagopal, 2005; Alicherry et al., 2005).

Obviously, unicast-based implementations are not readily applicable, or at least scalable enough to be employed in the one-to-many paradigm of a typical multicast communications setting. Moreover, given the bandwidth-constrained operation of wireless networks, existing wireline multicast solutions cannot be ported to mesh systems without fundamentally changing their behaviour to reduce overhead. Multicasting in MANETs and WSNs also addresses route recovery and energy concerns, which are characteristically different from the pivotal issues of throughput and interference raised in the middle-layer of MCMR WMNs. Routing in these networks is further complicated, given that the multiple radios on each node may dynamically switch on different channels. WMNbased multicasting has been discussed in Keegan et al. (2008), Ruiz and Gomez-skarmeta (2005), Roy et al. (2008), Zhao et al. (2006), Yuan et al. (2006), Shittu et al. (2008), Ruiz et al. (2006), Akyildiz and Wang (2008) and Crichigno et al. (2008), albeit for single-channel single-radio scenarios. The work in Karimi et al. (2010), on the other hand, targets multichannel single-radio settings, which characterise significantly different network configurations; further, the major emphasis in Karimi et al. (2010) is placed on throughput maximisation, of which multicast tree construction is not a necessity, 
as opposed to the problem of interest in this paper, which essentially narrows down to joint channel assignment and multicast tree construction with minimal interference.

In Gopinathan et al. (2009), multicast throughput optimisation in MCMR WMNs is modelled in terms of an Integer Linear Programming (ILP) formulation. To achieve the optimal result, the interplay between channel assignment in the MAC layer and multicast routing in the network layer should be accounted (Akyildiz and Wang, 2008); this has been neglected in Gopinathan et al. (2009). Moreover, unlike the case in our design, a multicast tree construction does not form the mainstay of Gopinathan et al. (2009).

In our brief review of the relevant literature, we specifically focus on studies addressing both channel assignment and multicast tree construction in MCMR WMNs. Existing schemes may be roughly classified into the following three categories: methods taking channel assignment for granted, thus treating multicast tree construction as the main problem (Nguyen and Nguyen, 2009); methods assuming a given multicast tree, thus solving for channel assignment as the core problem (Nguyen and Nguyen, 2008a, 2009; Yin et al., 2007); and finally, methods sequentially solving for both multicast tree construction and channel assignment (Zeng et al., 2007, 2010; Cheng and Yang, 2008a, 2008b, 2011; Lim et al., 2009).

In Zeng et al. (2007) and its extended version in Zeng et al. (2010), two methods for multicast tree construction and channel assignment in MCMR WMNs have been proposed. In the first method, mesh nodes are initially visited by conducting a BFS starting from the multicast source. This way, nodes are placed at different levels from source to multicast group members. Forwarding nodes in the multicast tree, on the other hand, are specified by taking on a bottomup approach: if each receiver node $v$ has several parents and one of their parents is on the multicast tree, this receiver is connected to that parent $\left(f_{v}\right)$. Otherwise, one of the parent nodes is selected randomly and one link is established to that parent $\left(f_{v}\right)$. The algorithm for node $f_{v}$ would continue recursively. After constructing the multicast tree, a so-called 'LCA' algorithm is used, which assigns channels to nodes depending on what level of BFS traversal tree they belong to. In particular, channel $i$ is assigned to nodes located at level $i$ of the tree. While the main advantage of this scheme lies in its simplicity, it also comes with the following shortcomings: when presented with multiple candidates, a multicast receiver has to be content with a parent chosen at random, which may not always work out to be the most promising choice. Also, there is no discussion as to which receiver node should initiate tree construction, despite the implications it might have on the specifics of the resultant tree structure. As for channel assignment, if the number of channels is more than the number of levels, the pool of available channels will be left underutilised.

The second method, namely 'MCM' also starts with placing the tree nodes at different levels using BFS. The minimum number of Relay Nodes (RNs), which form the multicast tree, would be determined according to the following approximation algorithm: parents can be chosen as
$\mathrm{RN}$ if one of their children has a fewer number of parents. When presented with a number of RNs, the node with the largest number of children is selected. Then, the elected $\mathrm{RN}$ together with its children is removed from the tree and the previous two steps would be repeated until all nodes at level $i+1$ are removed.

Channel assignment for ' $\mathrm{MCM}$ ', on the other hand, can be performed by either of the following two algorithms reported in Zeng et al. (2010): the top-down 'Ascending Channel Assignment' (ACA) assigns channels to levels starting from zero. When it runs out of channels, the algorithm re-assigns channel zero to the nodes of the next level and this process repeats. The shortcomings associated with ACA are as follows; under-utilisation may occur in case the number of sibling nodes is not equal in the whole tree. Also, ACA is prone to the hidden channel problem (Lim et al., 2009). Furthermore, for almost diagonal trees, ACA is subject to the same shortcomings as those of LCAs (Zeng et al., 2010). The second channel assignment scheme, referred to as 'Heuristic Channel Assignment' (HCA) draws on the channel separating conception, which indicates the disparity between two channel numbers. For example, the separation between channels 2 and 5 is 3 . In this method when a channel is assigned to node $u$, it should minimise the sum of squares of interference factor (Ding et al., 2008) between node $u$ and all nodes $v$ in its neighbourhood. However, since it has only account single-hop neighbours, the hidden channel problem may occur.

The hidden channel problem of Zeng et al. (2010) has motivated the work in Nguyen and Nguyen (2008), extended later in Nguyen and Nguyen (2009a). To reduce interference, a fitness function has been proposed to evaluate assignment of channel $c$ to node $v$. The hidden channel problem is dealt with by factoring the channel information of nodes within two hops into the objective function. However, it is assumed in Nguyen and Nguyen $(2008,2009$ a) that the multicast tree is readily available. It also relies on heavy broadcast message exchanges.

Another evaluation function is utilised in Yin et al. (2007); in particular, the assignment of channel $c$ to node $v$ is evaluated based on the probability of packet transmission by neighbouring nodes of $v$ on channel $c$. However, the specifics of the computation of this probability are not elaborated in Yin et al. (2007).

In Cheng and Yang (2008a, 2008b, 2011) three methods based on the GA, SA and the Tabu search technique have been proposed. In these methods, each multicast tree is represented by a two-dimensional array (chromosome), the rows of which determine a path from multicast source $S$ to the receiver $R_{i}$. Basically, a chromosome has $K$ rows for $K$ receivers. The number of columns in the chromosome is equal to the maximum path length from $S$ to the multicast group members. The representation of the chromosomes is based on the IDs of the nodes lying along the path from sender $S$ to the multicast group members. In this method, chromosomes are created as follows: the algorithm begins from the multicast source node $S$. A one-hop neighbour of $S$ is randomly selected and its ID is inserted into the chromosome. This process continues till it 
reaches a receiver $R_{i}$. Thus, a row of chromosomes is made. The same process is also run for the next receiver (within the next row). After constructing the multicast tree for each row of the chromosome, which is essentially a path from $S$ to $R_{i}$, the channels are sequentially assigned to the edges. The following disadvantages can be pointed out for these methods; first, assignment of the channels to each row of the chromosome is subject to similar shortcomings as those of the LCA algorithm; i.e., the hidden channel problem is not accounted for. Second, the nodes within the same level in the multicast tree are prone to interference. Finally, if the number of channels is more than the number of multicast tree levels, some channels will not be used at all.

The methods proposed in Zeng et al. (2007, 2010), Cheng and Yang (2008a, 2008b, 2011), Nguyen and Nguyen (2008, 2009a) and Yin et al. (2007) either construct a multicast tree or have assumed the tree is already constructed and then channels are assigned. In Nguyen and Nguyen (2009), however, the channel assignment is supposed to be done a priori and instead, the multicast tree should be constructed. More specifically, a centralised multicast tree algorithm, namely, 'Minimum Number of Transmission' (MCMNT), has been proposed, which aims to minimise the number of packets copied on to different channels in each node. The objective is to construct a tree with minimum cost using either the Dijkstra or the Bellman_Ford algorithm.

In Lim et al. (2009), a distributing bottom-up approach has been proposed to construct a multicast tree and to establish channel-radio associations. Initially, an approximation algorithm is used to generate the minimum RN set and to construct the multicast tree; each node identifies its two-hop neighbours with the least number of parents. Then, some of the parents of the two-hop neighbours with fewer children are candidate. Candidates with the best link quality will be selected. Afterwards, the selected nodes and all of their children are removed from the single-hop and two-hop nodes lists. This algorithm continues until both single-hop and two-hop neighbours' list are empty. However, it is subject to both the hidden channel problem and heavy broadcast message exchanges for placing nodes across levels.

Table 1 recapitulates our review of the existing methods with respect to their underlying ideas for both multicast tree construction and channel assignment. In sum, the major drawback with the existing methods is that they have considered channel assignment and multicast tree construction in the form of two independent issues and thus, have taken on an essentially sequential approach to a joint problem. In particular, the cross-interaction between multicasting at the network and channel assignment at the MAC layer is not accounted for in Zeng et al. (2007), Cheng and Yang (2008a, 2008b, 2011) and Lim et al. (2009) and in some methods, it is even assumed that the solution for these two sub-problems is pre-calculated (e.g., Nguyen and Nguyen (2008, 2009a, 2009b) and Yin et al. (2007). This is while the cross-layer design forms an integral part of a successful WMN-based implementation, as has been extensively and methodically argued in Peng et al. (2007). Moreover, all the reviewed schemes, except of course for
Lim et al. (2009), are built around a centralised perspective and their heuristic mentality is essentially incapable of providing the optimal solution.

Table 1 Existing multicast designs for Multi-Channel Multi-Radio Wireless Mesh Networks

\begin{tabular}{|c|c|c|}
\hline Ref. & $\begin{array}{l}\text { Multicast tree } \\
\text { construction }\end{array}$ & Channel assignment \\
\hline \multirow[t]{2}{*}{$\begin{array}{l}\text { Zeng et al. (2007, } \\
2010)\end{array}$} & $\begin{array}{l}\text { A centralised heuristic } \\
\text { bottom-up algorithm } \\
\text { which utilises BFS }\end{array}$ & $\begin{array}{l}\text { LCA: An ascending } \\
\text { method }\end{array}$ \\
\hline & $\begin{array}{l}\text { MCM: A centralised } \\
\text { approximate top-down } \\
\text { algorithm }\end{array}$ & $\begin{array}{l}\text { An ascending method } \\
\text { A heuristic method } \\
\text { based on channel } \\
\text { separation which only } \\
\text { considers single-hop } \\
\text { interference }\end{array}$ \\
\hline $\begin{array}{l}\text { Nguyen and } \\
\text { Nguyen (2008, } \\
\text { 2009a) }\end{array}$ & $\begin{array}{l}\text { Multicast tree is } \\
\text { assumed to be } \\
\text { constructed a priori }\end{array}$ & $\begin{array}{l}\text { M4: An improved } \\
\text { version of Zeng et al. } \\
\text { (2010), which also } \\
\text { considers two-hop } \\
\text { interference }\end{array}$ \\
\hline Yin et al. (2007) & $\begin{array}{l}\text { Multicast tree is } \\
\text { assumed to be } \\
\text { constructed a priori }\end{array}$ & $\begin{array}{l}\text { A greedy channel } \\
\text { assignment that } \\
\text { utilises BFS }\end{array}$ \\
\hline $\begin{array}{l}\text { Cheng and Yang } \\
(2008 \mathrm{a}, 2011)\end{array}$ & $\begin{array}{l}\text { A centralised scheme } \\
\text { based on GA, SA and } \\
\text { TS techniques }\end{array}$ & An ascending method \\
\hline $\begin{array}{l}\text { Nguyen and } \\
\text { Nguyen (2009b) }\end{array}$ & $\begin{array}{l}\text { MCMNT: A heuristic } \\
\text { method for computing } \\
\text { edge cost, coupled } \\
\text { with minimum cost } \\
\text { tree construction using } \\
\text { either the Dijkstra } \\
\text { or the Bellman-Ford } \\
\text { algorithms }\end{array}$ & $\begin{array}{l}\text { Channel assignment } \\
\text { is assumed to be } \\
\text { performed a priori }\end{array}$ \\
\hline Lim et al. (2009) & $\begin{array}{l}\text { An improved version } \\
\text { of MCM which also } \\
\text { factors in the link } \\
\text { quality }\end{array}$ & $\begin{array}{l}\text { A heuristic } \\
\text { algorithm }\end{array}$ \\
\hline
\end{tabular}

Considering the above weaknesses, in what follows, we mainly focus on achieving the optimal solution for joint channel assignment and the multicast tree construction problem in MCMR WMNs. For this, we present two mathematical BIP-based formulations which are inherently centralised. The former solves the problem via a cross-layer design and the latter is a layered protocol which provides the optimal solution for each sub-problem.

\section{Mathematical framework}

The mathematical framework presented in this paper is basically a BIP formulation, which, as opposed to the existing heuristic- or meta-heuristic-based schemes, guarantees the optimality of the solution. Given a problem definition in terms of a set of linear equality/inequality constraints, a BIP model, as a variant of linear programming, determines a way to obtain the best feasible solution. From the geometric viewpoint, a feasible region, in the form of a convex polyhedron, is 
defined with linear constraints. Within this region, provided that a feasible solution exists and also under the condition that the linear objective function is bounded, the optimum result is always achievable on the boundary of optimal levelset by the maximum/minimum principle for convex/concave functions (Vandenberghe, 2004). Given the wholeness and properness of the set of constraints formulating the problem of interest, BIP guarantees global optimality of the resulting solution.

\subsection{System model and assumptions}

In this section, we present the assumptions of our mathematical formulations for the problem of multicast tree construction with minimal interference. We have formulated our BIP models to find the MCT-based multicast tree. As for interference computation, we have adopted the protocol model described in Hossain and Leung (2007) and Crichigno et al. (2008). In this model, a given transmission from a node Src to a node Des is said to be successful only if:

- the nodes' distance is less than the transmission range

- no third node, located within the interference range of the receiving node Des, is transmitting.

The adopted model can further be refined to comply with IEEE 802.11-style MAC protocols; i.e., the sending node Src is also required to be free of interference, as it needs to receive the link layer acknowledgement from the receiving node Des. Specifically, any node Temp, which is within the interference range of Src or Des, should not be transmitting.

Other assumptions include: all mesh routers are distributed randomly on a plane. Each router is equipped with multiple radio interfaces and the number of radios is not more than that of the available non-overlapping channels. All radio interfaces on wireless routers make use of omni-directional antennas and have identical transmission/interference ranges.

\subsection{Proposed methods}

As previously mentioned, in the existing methods the crossinteraction between multicast at the network and channel assignment at the MAC layer has not been considered. Therefore, in this section, we present a BIP-based formulation in which both multicast tree construction and channel assignment problems are solved conjointly. We called this method the 'cross-layer' design. Afterwards, we will propose another formulation, namely the 'Sequential' design, in which the optimal solution for each sub-problem is provided.

Before we present the formulations, common definitions for both methods should be described. Tables 2 and 3 show the common defined sets and parameters for both proposed sequential and cross-layer methods. Necessary descriptions are included in the tables too.

\subsubsection{Proposed cross-layered method}

In this section, the required variables used within the BIP cross-layer model followed by the defined constraints are described.
Table 2 Common defined sets for both sequential and cross-layer methods

Nodes $=\left\{N_{1}, N_{2}, N_{3}, \ldots, N_{n}\right\}$

ChannelList $=\left\{C_{1}, C_{2}, C_{3}, \ldots, C_{c}\right\}$

mesh routers

MulticastSource $=\left\{N_{i}\right\}, N_{i} \in$ Nodes

channels

MulticastTarget $=\{T, T, T, T\}, T \in$ Nodes multicast targets

Radio $=\left\{R_{1}, R_{2}, R_{3}, \ldots, R_{R}\right\}$

$R\left(N_{i}\right), N_{i} \in$ Nodes

mesh routers', radios node $N_{i}^{\prime}$ s radios

Table 3 Common predefined parameters for both Sequential and cross-layer methods

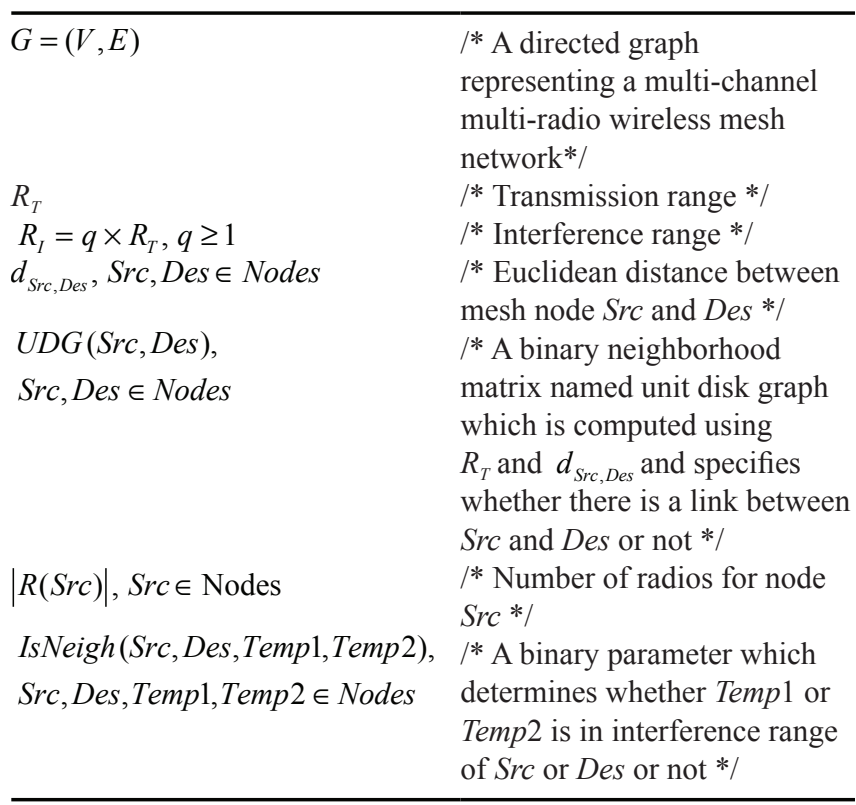

\subsubsection{Defined variables}

Before presenting the BIP cross-layer model for the joint multicast tree construction and channel assignment problem, necessary variables should be introduced. In what follows, the variables are discussed.

A binaryvariable $F_{C_{i, S R, S r c}}$ where $S r c \in$ Nodes, SR $\in$ Radios and $C_{i} \in$ ChannelList is defined to determine whether or not the channel $C_{i}$ is assigned to radio $S R$ of node $S r c$.

A binary variable $\operatorname{Link}\left(\operatorname{Src}, S R, D e s, D R, C_{i}\right)$ such that Src, Des $\in$ Nodes, SR, DR $\in$ Radios and $C_{i} \in$ ChannelList is defined to determine whether or not a link between radio $S R$ of node $S r c$ and radio $D R$ of node Des is established on channel $C_{i}$.

Two non-negative variables InputLink $(\operatorname{Src})$ and OutputLink(Src) with the following definitions are required to determine the number of incoming/outgoing links to/from mesh router $S r c$, respectively.

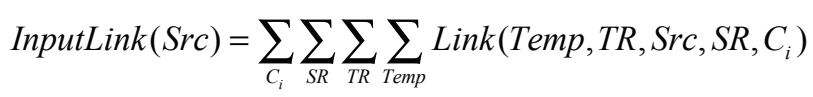

$$
\text { OutputLink }(\operatorname{Src})=\sum_{C_{i}} \sum_{S R} \sum_{T R} \sum_{\text {Temp }} \operatorname{Link}\left(\operatorname{Src}, S R, T e m p, T R, C_{i}\right)
$$


where Src,Temp $\in$ Nodes, TR, SR $\in$ Radios, $C_{i} \in$ ChannelList.

Also, a non-negative variable Interference $(S r c, S R, D e s$, $D R, C_{i}$ ) such that Src, Des $\in$ Nodes, $S R, D R \in$ Radios, $C_{i} \in$ ChannelList is required to determine the number of interfering links with link $\left(\operatorname{Src}, S R, \operatorname{Des}, D R, C_{i}\right)$.

SIL is another non-negative variable which stands for Sum of Interfering Links and computes the number of links within the interference range of Src or Des on channel $C_{i}$. This variable is mainly introduced to account for the hidden channel problem:

$$
\begin{aligned}
\operatorname{SIL}\left(\operatorname{Src}, S R, \operatorname{Des}, D R, C_{i}\right) \\
=\sum_{\text {Temp } 2} \sum_{T R 2} \sum_{\text {Temp } 1} \sum_{T R 1}[\operatorname{IsNeigh}(\operatorname{Src}, \text { Des }, \text { Temp } 1, \text { Temp } 2) \\
\quad \times \operatorname{Link}\left(\operatorname{Temp} 1, T R 1, \operatorname{Temp} 2, T R 2, C_{i}\right) \\
\left.\quad-\operatorname{Link}\left(\operatorname{Src}, \operatorname{SR}, \operatorname{Des}, D R, C_{i}\right)\right]
\end{aligned}
$$

where Src, Des $\in$ Temp 1, Temp2, $\in$ Nodes, SR, DR, TR1, TR2 $\in$ Radios, $C_{i} \in$ ChannelList.

TotalInterference is a non-negative variable which determines total interference across the multicast tree:

\section{Totalinterference}

$$
=\sum_{D e s} \sum_{D R} \sum_{S r c} \sum_{S R} \sum_{C_{i}} \text { Interference }\left(S r c, S R, D e s, D R, C_{i}\right)
$$

where Src, Des $\in$ Nodes, $S R, D R \in$ Radios, $C_{i} \in$ ChannelList.

Finally we define a non-negative variable which determines the number of links forming the multicast tree:

$$
\begin{aligned}
& \text { TotalLinks } \\
& =\sum_{D e s} \sum_{D R} \sum_{S r c} \sum_{S R} \sum_{C_{i}} \operatorname{Link}\left(\operatorname{Src}, S R, D e s, D R, C_{i}\right)
\end{aligned}
$$

where Src,Des $\in$ Nodes, $S R, D R \in$ Radios, $C_{i} \in$ ChannelList.

\subsubsection{Defined constraints}

In what follows the required constraints in cross-layer design are introduced.

From the multicast tree definition, the number of all incoming links to a node except for the multicast source and multicast target should be at most 1 . Constraint (6) satisfies this property.

$$
\operatorname{InputLink}(\operatorname{Src}) \leq 1 \text {, }
$$

Src $\in$ Nodes $\backslash$ (MulticastSource, MulticastTarget).

As is shown in constraint (7), in a multicast tree with multi-radio nodes, the number of all incoming links to and outgoing links from a node except for the multicast source and multicast target should be at most equal to the number of radios per each node.

$$
\begin{aligned}
& \text { InputLink }(\operatorname{Src})+\text { OutputLink }(\operatorname{Src}) \leq|R(\operatorname{Src})|, \\
& \text { Src } \in \text { Nodes } \backslash \text { (MulticastSource, MulticastTarget }) .
\end{aligned}
$$

It is obvious that there are no incoming links to a multicast source. Constraint (8) ensures this feature.

$$
\operatorname{InputLink}(\operatorname{Src})=0 \text {, }
$$

Src $\in$ MulticastSource.
In a multicast tree, as shown in constraint (9), the number of outgoing links from the multicast source should be at most equal to the number of multicast targets.

$$
\text { OutputLink }(\text { Src }) \leq \mid \text { MulticastTarget } \mid \text {, }
$$$$
\text { Src } \in \text { MulticastSource. }
$$

The multicast source must have at least one outgoing link. Constraint (10) guarantees this property.

$$
\begin{aligned}
& \text { OutputLink }(\text { Src }) \geq 1, \\
& \text { Src } \in \text { MulticastSource. }
\end{aligned}
$$

Also, all multicast targets should have just one incoming link. Equality constraint (11) ensures this characteristic.

$$
\begin{aligned}
& \text { InputLink }(\operatorname{Src})=1, \\
& \text { Src } \in \text { MulticastTarget. }
\end{aligned}
$$

Similarly, the number of outgoing links from a multicast target should be exactly zero (constraint 12).

$$
\begin{aligned}
& \text { OutputLink }(\operatorname{Src})=0, \\
& \text { Src } \in \text { MulticastTarget. }
\end{aligned}
$$

In MCMR WMNs, every radio of a node should be used at most once for either input or output purposes. Constraint (13) implements this feature.

$$
\begin{aligned}
& \sum_{\text {Des }} \sum_{D R} \sum_{C_{i}} \operatorname{Link}\left(\operatorname{Src}, S R, \text { Des, DR, } C_{i}\right) \\
& \quad+\sum_{\text {Des }} \sum_{D R} \sum_{C_{i}} \operatorname{Link}\left(\operatorname{Des}, D R, S r c, S R, C_{i}\right) \leq 1 \\
& \quad \text { Src }, \text { Des } \in \text { Nodes, SR,DR } \in \text { Radios }, C_{i} \in \text { ChannelList } .
\end{aligned}
$$

Inequalities (14) and (15), together, define an if and only if' constraint; i.e., if a node has an incoming edge, then it definitely has an outgoing edge as well and vice versa.

OutputLink $(\operatorname{Src}) \geq \operatorname{InputLink}(\operatorname{Src}), \quad \operatorname{Src} \in$ Nodes

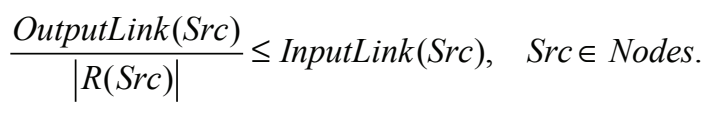

In MCMR WMNs, every channel $C_{i}$ should be assigned to radios of a node at most once. Inequality constraint (16) guarantees this property.

$$
\begin{aligned}
& \sum_{\text {Des }} \sum_{D R} \sum_{S R} \operatorname{Link}\left(\operatorname{Src}, S R, \text { Des }, D R, C_{i}\right) \\
& +\sum_{D e s} \sum_{D R} \sum_{S R} \operatorname{Link}\left(\operatorname{Des}, D R, S r c, S R, C_{i}\right) \leq 1 \\
& \text { Src, Des } \in \text { Nodes, SR,DR } \in \text { Radios, } \\
& \quad C_{i} \in \text { ChannelList. }
\end{aligned}
$$

The aforementioned variable Interference $\left(\operatorname{Src}, S R\right.$, Des, $\left.D R, C_{i}\right)$ is computed as equation (17).

$$
\begin{aligned}
& \text { Interference }\left(\operatorname{Src}, S R, D e s, D R, C_{i}\right) \\
& =\operatorname{Link}\left(\operatorname{Src}, S R, D e s, D R, C_{i}\right) \times \operatorname{SIL}\left(\operatorname{Src}, S R, \operatorname{Des}, D R, C_{i}\right) .
\end{aligned}
$$


Thus, if $\operatorname{Link}\left(\operatorname{Src}, S R, \operatorname{Des}, D R, C_{i}\right)$ is established, then its interference is equal to the variable SIL; otherwise, it is zero. Constraints (18)-(20) correspond to the multiplication of the two variables Link and SIL in LP terms.

$$
\begin{aligned}
& \text { Interference }\left(\operatorname{Src}, S R, \text { Des, DR, } C_{i}\right) \leq(\mid \text { Nodes } \mid \\
& \left.\quad \times(\mid \text { Nodes } \mid-1) / 2 \times \operatorname{Link}\left(\operatorname{Src}, S R, D e s, D R, C_{i}\right)\right)
\end{aligned}
$$

$$
\begin{aligned}
& \text { Interference }\left(\operatorname{Src}, S R, \text { Des, DR, } C_{i}\right) \\
& \quad \leq \operatorname{SIL}\left(\operatorname{Src}, \operatorname{SR}, \operatorname{Des}, D R, C_{i}\right)
\end{aligned}
$$

Interference $\left(\operatorname{Src}, S R\right.$, Des, DR, $\left.C_{i}\right)$

$$
\geq \operatorname{SIL}\left(\operatorname{Src}, S R, \operatorname{Des}, D R, C_{i}\right)
$$$$
-\left(\mid \text { Nodes } \mid \times \frac{\mid \text { Nodes } \mid-1}{2}\right)
$$

$\times\left(1-\operatorname{Link}\left(\operatorname{Src}, S R, \operatorname{Des}, D R, C_{i}\right)\right)$

Src, Des $\in$ Nodes, $S R, D R \in$ Radios,

$C_{i} \in$ ChannelList.

Constraints (21) and (22) stipulate the link establishment conditions between two nodes $\mathrm{Src}$ and Des:

- they are located within the transmission range of each other

- a common channel is assigned to both radio $S R$ of $S r c$ and radio $D R$ of Des.

$$
\begin{aligned}
& \quad \operatorname{Link}\left(\operatorname{Src}, S R, D e s, D R, C_{i}\right) \times d_{S r c, D e s} \\
& \quad \leq \operatorname{Link}\left(\operatorname{Src}, S R, D e s, D R, C_{i}\right) \times R_{T} \\
& \operatorname{Link}\left(\operatorname{Src}, S R, D e s, D R, C_{i}\right) \times F_{C_{i, S R, S r c}} \\
& \quad=\operatorname{Link}\left(\operatorname{Src}, S R, D e s, D R, C_{i}\right) \times F_{C_{i, D R, D e s}}
\end{aligned}
$$

where Scr, Des $\in$ Nodes, $S R, D R \in$ Radios, $C_{i} \in$ ChannelList.

Finally, since the interference is computed from the established links, the objective of this model can be defined:

\section{Minimise (TotalLinks +TotalInterference).}

Altogether, the BIP formulation for cross-layer design is modelled as follows:

Minimise (TotalLinks +TotalInterference)

Subject to:

\begin{tabular}{l}
\hline InputLink $($ Src $) \leq 1, \quad$ Src $\in$ Nodes $\backslash($ MulticastSource, \\
MulticastTarget $)$
\end{tabular}

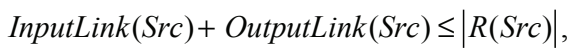

Src $\in$ Nodes $\backslash$ (MulticastSource, MulticastTarget)

InputLink $(\operatorname{Src})=0, \quad$ Src $\in$ MulticastSource

OutputLink $(\operatorname{Src}) \leq \mid$ MulticastSource $\mid, \quad$ Src $\in$ MulticastSource

OutputLink $(S r c) \geq 1, \quad$ Src $\in$ MulticastSource
$\operatorname{InputLink}(\operatorname{Src})=1, \quad$ Src $\in$ MulticastTarget

OutputLink $(\operatorname{Src})=0, \quad$ Src $\in$ MulticastTarget

$\sum_{D e s} \sum_{D R} \sum_{C_{i}} \operatorname{Link}\left(\operatorname{Src}, S R, D e s, D R, C_{i}\right)$

$+\sum_{\text {Des }} \sum_{D R} \sum_{C_{i}} \operatorname{Link}\left(\operatorname{Des}, D R, S r c, S R, C_{i}\right) \leq 1$,

Src, Des $\in$ Nodes, $S R, D R \in$ Radios, $C_{i} \in$ ChannelList

OutputLink $(\operatorname{Src}) \geq \operatorname{InputLink}(\operatorname{Src})$

$\frac{\text { OutputLink }(\operatorname{Src})}{|R(\operatorname{Src})|} \leq \operatorname{InputLink}(\operatorname{Src}), \operatorname{Src} \in$ Nodes

$\sum_{D e s} \sum_{D R} \sum_{S R} \operatorname{Link}\left(\operatorname{Src}, S R, D e s, D R, C_{i}\right)$

$+\sum_{D e s} \sum_{D R} \sum_{S R} \operatorname{Link}\left(\operatorname{Des}, D R, S r c, S R, C_{i}\right) \leq 1$,

Src, Des $\in$ Nodes, $S R, D R \in$ Radios, $C_{i} \in$ ChannelList

Interference $\left(\operatorname{Src}, S R\right.$, Des, DR, $\left.C_{i}\right) \leq(\mid$ Nodes $\mid \times(\mid$ Nodes $\mid-1) / 2)$

$\times \operatorname{Link}\left(\operatorname{Src}, S R, \operatorname{Des}, D R, C_{i}\right)$

Interference $\left(\operatorname{Src}, S R, D e s, D R, C_{i}\right) \leq \operatorname{SIL}\left(\operatorname{Src}, S R, D e s, D R, C_{i}\right)$

Interference $\left(S r c, S R, D e s, D R, C_{i}\right) \geq \operatorname{SIL}\left(\operatorname{Src}, S R, D e s, D R, C_{i}\right)$

$-(\mid$ Nodes $\mid \times(\mid$ Nodes $\mid-1) / 2) \times\left(1-\operatorname{Link}\left(\operatorname{Src}, S R, D e s, D R, C_{i}\right)\right)$

Src, Des $\in$ Nodes, $\quad S R, D R \in$ Radios, $C_{i} \in$ ChannelList

$\operatorname{Link}\left(\operatorname{Src}, S R, D e s, D R, C_{i}\right) \times d_{\text {Src,Des }} \leq \operatorname{Link}\left(\operatorname{Src}, S R, D e s, D R, C_{i}\right) \times R_{T}$

$\operatorname{Link}\left(\operatorname{Src}, S R, D e s, D R, C_{i}\right) \times F_{C_{i, S R, S t r}}$

$=\operatorname{Link}\left(\operatorname{Src}, S R, \operatorname{Des}, D R, C_{i}\right) \times F_{C_{i, D R}, D e s}$

Src, Des $\in$ Nodes, SR,DR $\in$ Radios, $C_{i} \in$ ChannelList

\subsubsection{Proposed Layered method}

In this design, say Sequential method, we model the overall problem as two separated sub-problems which are solved in sequence. Clearly, in the first phase of the Sequential method, a MCT-based optimal multicast tree is constructed. Afterwards, the resultant tree constructed from the first phase is fed to the channel assignment phase as input.

Hereafter, we refer to the multicast tree construction model as the MT_Model. Similarly, the channel assignment model is referred to as the CA_Model. The algorithm of the layered design is shown in Figure 1. In two sub-sections 3.2.2.1 and 3.2.2.2, two sub-problems of this approach are discussed in detail.

Figure 1 Algorithm of the proposed Sequential method

1. Preprocessing Phase
1.2 Defining the required Sets presented in table 2
1.3 Defining the required Parameters presented in table 3
2. Solving Phase
2.1 Solve MT_Model
2.2 Compute parameter Link_(Src,Des) using output of MT_Model and
consider it as input to CA_Model
2.3 Solve CA_Model




\subsubsection{Multicast tree construction sub-problem}

\subsection{Defined variables}

In this section, the required variables within the MT_Model of the proposed Sequential method are introduced as follows:

A binary variable is needed to indicate whether there is a link between radio $S R$ of node $S r c$ and radio $D R$ of node Des or not. We define this variable as:

Link (Src,SR,Des, DR), Src, Des $\in$ Nodes, DR, SR $\in$ Radios.

Also, a non-negative variable which determines the numbers of incoming links to every mesh router is required. For this, we define the variable InputLink(Src) with the equation (23).

$$
\begin{aligned}
& \operatorname{InputLink}(\operatorname{Src})=\sum_{S R} \sum_{D e s} \sum_{D R} \operatorname{Link}(\operatorname{Des}, D R, S r c, S R) \\
& \text { Src, Des } \in \text { Nodes, DR, SR } \in \text { Radios }
\end{aligned}
$$

Similarly, we need another non-negative variable which determines the number of outgoing links from every mesh router. Thus, we define the variable OutputLink $(S r c)$ with definition (24).

$$
\begin{gathered}
\text { OutputLink }(\operatorname{Src})=\sum_{S R} \sum_{D e s} \sum_{D R} \operatorname{Link}(\operatorname{Src}, S R, D e s, D R), \\
\text { Src,Des } \in \text { Nodes, DR, SR } \in \text { Radios }
\end{gathered}
$$

Finally, the non-negative variable Total_Link is required to determine the total number of links forming the multicast tree:

$$
\begin{aligned}
\text { Total_Link } & =\sum_{\text {Src }} \sum_{S R} \sum_{D e s} \sum_{D R} \operatorname{Link}(\operatorname{Src}, S R, \text { Des, DR }), \\
\text { Src, Des } & \in \text { Nodes, DR, SR } \in \text { Radios. }
\end{aligned}
$$

\subsection{Defined constraints}

In this sub-section, the required constraints for the multicast tree construction sub-problem of the proposed Sequential method are discussed;

In case of the multicast tree, the number of all the input links to a node except multicast source and multicast target should be at most 1. Constraint (26) ensures this property.

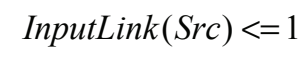

Src $\in$ Nodes $\backslash$ (MulticastSource,MulticastTarget).

In MCMR WMNs, the sum of all incoming links to and outgoing links from a node except multicast source and multicast target should be at most equal the number of radios. Constraint (27) guarantees this feature.

$$
\begin{aligned}
& \text { InputLink }(\operatorname{Src})+\text { OutPutLink }(\operatorname{Src})<=|R(\operatorname{Src})|, \\
& \text { Src } \in \text { Nodes } \backslash(\text { MulticastSource, MulticastTarget }) .
\end{aligned}
$$

In MCMR WMNs, if two nodes are located in the transmission range of each other, then one link can be established between them (constraint (28)).

$$
\begin{gathered}
\operatorname{Link}(\operatorname{Src}, S R, \text { Des }, D R)<=U D G(\operatorname{Src}, \text { Des }), \\
\text { Src, Des } \in \text { Nodes, DR, SR } \in \text { Radios. }
\end{gathered}
$$

From the multicast tree definition, the multicast source node has no incoming links. The equality constraint (29) satisfies this property.

$$
\operatorname{InputLink}(\operatorname{Src})=0, \quad \text { Src } \in \text { MulticastSource }
$$

Similarly, the number of outgoing links from the multicast source node should be at most equal to the number of multicast targets. This feature is ensured by constraint (30).

$$
\text { OutputLink }(\operatorname{Src})<=\mid \text { MulticastTarget } \mid \text {, }
$$$$
\text { Src } \in \text { MulticastSource. }
$$

Furthermore, the number of outgoing links from multicast source node should be at least one(constraint 31).

$$
\text { OutputLink }(\operatorname{Src})>=1, \quad \text { Src } \in \text { MulticastSource }
$$

The number of incoming links to every multicast target should be exactly one:

$$
\operatorname{InputLink}(\operatorname{Src})=1, \quad \operatorname{Src} \in \text { MulticastTarget. }
$$

Equality constraint (33) ensures the multicast targets have no outgoing links.

$$
\text { OutputLink }(\operatorname{Src})=0, \quad \text { Src } \in \text { MulticastTarget } .
$$

The proposed formulation should occur with guarantees of one-hop loops in the multicast tree. Constraint (34) ensures this inherent feature of the constructed tree.

$$
\begin{aligned}
U D G(\operatorname{Src}, \text { Temp }) \times( & \sum_{S R} \sum_{T R} \operatorname{Link}(\operatorname{Src}, \text { SR,Temp }, T R) \\
& +\sum_{S R} \sum_{T R} \operatorname{Link}(\operatorname{Temp}, T R, S r c, S R) \leq 1 \\
& \times \text { UDG }(\operatorname{Src}, \text { Temp }) \\
& \text { Src, Temp } \in \text { Nodes }, T R, S R \in \text { Radios. }
\end{aligned}
$$

In MCMR WMNs, every radio of a node has to form at most one link:

$$
\begin{aligned}
U D G(\operatorname{Src}, \text { Des }) \times( & \sum_{\text {Des }} \sum_{D R} \operatorname{Link}(\operatorname{Src}, S R, D e s, D R) \\
& +\sum_{\text {Des }} \sum_{D R} \operatorname{Link}(\operatorname{Des}, D R, S r c, S R) \leq 1 \\
& \times U D G(\operatorname{Src}, D e s) .
\end{aligned}
$$

Constraints (36) and (37) explain an 'if and only if' condition. That is, if a node has one or more outgoing links, then it should have an incoming link and also if it has an incoming link, then it will have at least one outgoing link:

$$
\begin{aligned}
& \text { OutputLink }(\operatorname{Src})>=\text { InputLink }(\text { Src }), \\
& \text { Src } \in \text { Nodes } \backslash(\text { MulticastSource, MulticastTarget }) \\
& \frac{\text { OutputLink }(\operatorname{Src})}{\mid \text { radios } \mid}<=\text { InputLink }(\text { Src }), \\
& \text { Src } \in \text { Nodes } \backslash \text { (MulticastSource, MulticastTarget). }
\end{aligned}
$$


And finally, the objective of the multicast tree construction sub-problem is:

\section{MinimiseTotal_Link.}

The BIP model for this sub-problem of the proposed layered design is summarised as follows:

\section{MinimiseTotal_Link}

Subject to:

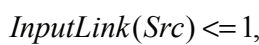

Src $\in$ Nodes $\backslash$ (MulticastSource, MulticastTarget)

$(\operatorname{InputLink}(\operatorname{Src})+$ OutPutLink $(\operatorname{Src}))<=|R(\operatorname{Src})|$,

Src $\in$ Nodes $\backslash$ (MulticastSource, MulticastTarget)

$\operatorname{Link}(\operatorname{Src}, S R, D e s, D R)<=U D G(\operatorname{Src}, D e s)$,

Src, Des $\in$ Nodes, DR, $S R \in$ Radios

$\operatorname{InputLink}(\operatorname{Src})=0, \operatorname{Src} \in$ MulticastSource

OutputLink $($ Src $)<=\mid$ MulticastTarget $\mid$, Src $\in$ MulticastSource

OutputLink $(\operatorname{Src})>=1$, Src $\in$ MulticastSource

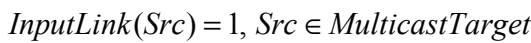

OutputLink $(\operatorname{Src})=0$, Src $\in$ MulticastTarget

$U D G(\operatorname{Src}, T e m p) \times\left(\sum_{S R} \sum_{T R} \operatorname{Link}(\operatorname{Src}, S R, T e m p, T R)\right.$

$$
\begin{aligned}
& \left.+\sum_{S R} \sum_{T R} \operatorname{Link}(\text { Temp }, T R, S r c, S R)\right) \leq 1 \\
& \times U D G(\text { Src }, \text { Temp }), \\
& \text { Src,Temp } \in \text { Nodes, TR, SR } \in \text { Radios }
\end{aligned}
$$

$$
\begin{aligned}
U D G(\operatorname{Src}, D e s) \times( & \sum_{\text {Des }} \sum_{D R} \operatorname{Link}(\operatorname{Src}, S R, D e s, D R) \\
& \left.+\sum_{D e s} \sum_{D R} \operatorname{Link}(\operatorname{Des}, D R, S r c, S R)\right) \leq 1 \\
& \times U D G(\operatorname{Src}, D e s)
\end{aligned}
$$

OutPutLink $(\operatorname{Src})>=\operatorname{InputLink}(\operatorname{Src})$,

Src $\in$ Nodes $\backslash$ (MulticastSource, MulticastTarget)

$\frac{\text { OutPutLink }(\operatorname{Src})}{\mid \text { radios } \mid}<=\operatorname{InputLink}(\operatorname{Src})$,

Src $\in$ Nodes $\backslash$ (MulticastSource, MulticastTarget)

\subsubsection{Channel assignment sub-problem}

After solving the MT_Model and the CA_Model, here it is necessary to define a binary parameter Link_which is computed using the binary variable $\operatorname{Link}(\operatorname{Src}, D e s)$ as output of the first phase (MT_Model). The parameter Link is set to one if $\operatorname{Link}(\operatorname{Src}, \mathrm{Des})$ has been established and zero otherwise.

\subsection{Defined variables}

We define four variables within the CA_Model of the proposed Sequential design as follows:

A non-negative variable Sum of Interfering Links $(S I L)$ is needed to compute the number of links within the interference range of the source or destination of a link:

$$
\begin{aligned}
\operatorname{SIL}\left(\operatorname{Src}, \text { Des }, C_{i}\right) & =\operatorname{Link} \_(\operatorname{Src}, \text { Des }) \\
& \times \sum_{\text {Temp } 1 \text { Temp } 2}(\operatorname{IsNeigh}(\operatorname{Src}, \text { Des }, \text { Temp } 1, \text { Temp } 2) \\
& \times \operatorname{Link} \_(\text {Temp } 1, \text { Temp } 2) \\
& \left.\times \operatorname{Assign}\left(\operatorname{Temp} 1, \text { Temp } 2, C_{i}\right)\right) \\
& \left.-\operatorname{Assign}\left(\operatorname{Src}, \text { Des }, C_{i}\right)\right)
\end{aligned}
$$

where Src, Des,Temp1,Temp $2 \in$ Nodes, $C_{i} \in$ ChannelList.

This variable considers the hidden channel problem as well.

A binary variable $\operatorname{Assign}\left(\operatorname{Src}, \operatorname{Des}, C_{i}\right)$ such as $\operatorname{Src}, \operatorname{Des} \in$ Nodes and $C_{i} \in$ ChannelList, is needed to determine whether $C_{i}$ is assigned to the established link between two nodes $\mathrm{Src}$, Des or not.

The non-negative variable Interference $\left(\operatorname{Src}, \operatorname{Des}, C_{i}\right)$, denotes the number of interfering links with link ( $S r c$, Des, $C_{i}$ ) and is computed as equation (39).

$$
\begin{aligned}
& \text { Interference }\left(\operatorname{Src}, \text { Des, } C_{i}\right) \\
& \quad=\operatorname{Assign}\left(\operatorname{Src}, \operatorname{Des}, C_{i}\right) \times \operatorname{SIL}\left(\operatorname{Src}, \operatorname{Des}, C_{i}\right) .
\end{aligned}
$$

Finally, the non-negative variable Total_Interference is defined to compute the overall interference in the multicast tree:

$$
\begin{array}{r}
\text { Total_Interference }=\sum_{\text {Src Des }} \sum_{C_{i}} \sum_{\text {Interference }\left(\text { Src }, \text { Des }, C_{i}\right)} \\
\text { Src, Des } \in \text { Nodes }, C_{i} \in \text { ChannelList }
\end{array}
$$

\subsection{Defined constraints}

The required constraints for the CA_Model of the proposed Sequential method are discussed as follows:

Just one channel should be assigned to every link in MCMR WMNs (constraint (41)).

$$
\begin{gathered}
\left.\sum_{C_{i}} \operatorname{Assign}\left(\operatorname{Src}, \text { Des }, C_{i}\right)=\text { Link_(Src,Des }\right), \\
\operatorname{Src}, \text { Des } \in \text { Nodes }, C_{i} \in \text { ChannelList }
\end{gathered}
$$

If a link is established and channel $C_{i}$ is assigned to it, then its interference is equal to variable SIL; otherwise, it is zero. Constraints (42)-(44), correspond to the multiplication of the two variables Link and SIL in LP terms.

$U D G(\operatorname{Src}$, Des $) \times \operatorname{Link} \_(\operatorname{Src}, D e s) \times \operatorname{Interference}\left(\operatorname{Src}, D e s, C_{i}\right)$

$$
\begin{aligned}
& \leq\left(\left(\sum_{\text {temp } 1} U D G(\text { Src }, \text { temp } 1) \times\left(\sum_{\text {temp } 1} U D G(\operatorname{Src}, \text { temp } 1)-1\right)\right)\right. \\
& \left.+\left(\sum_{\text {temp } 2} U D G(\text { Des }, \text { temp } 2) \times\left(\sum_{\text {temp } 2} U D G(\text { Des }, \text { temp } 2)-1\right)\right)\right) \\
& \left.\times \operatorname{Assign}\left(\operatorname{Src}, \text { Des }, C_{i}\right) \times U D G(\operatorname{Src}, \text { Des }) \times \text { Link_(Src }, \text { Des }\right)
\end{aligned}
$$




$$
\begin{aligned}
& U D G(\operatorname{Src}, \text { Des }) \times \text { Link_ }(\operatorname{Src}, \text { Des }) \\
& \times \text { Interference }\left(\operatorname{Src}, \text { Des, } C_{i}\right) \leq \operatorname{SIL}\left(\operatorname{Src}, \text { Des }, C_{i}\right) \\
& \times U D G(\operatorname{Src}, \text { Des }) \times \text { Link_(Src,Des }) \text {, } \\
& \text { Src, Des } \in \text { Nodes, } C_{i} \in \text { ChannelList } \\
& U D G(\operatorname{Src}, \text { Des }) \times \text { Link_}_{-}(\operatorname{Src}, \text { Des }) \times \text { Interference }\left(\operatorname{Src}, \text { Des }, C_{i}\right) \\
& \geq\left(\operatorname{SIL}\left(\operatorname{Src}, \text { Des }, C_{i}\right)-\left(\sum_{\text {temp } 1} U D G(\operatorname{Src}, \text { temp } 1)\right.\right. \\
& \left.\times\left(\sum_{\text {temp } 1} U D G(\operatorname{Src}, \text { temp } 1)-1\right)\right)+\left(\sum_{\text {temp } 2} U D G(\text { Des, temp } 2)\right. \\
& \left.\left.\times\left(\sum_{\text {temp } 2} U D G(\text { Des, temp } 2)-1\right)\right)\right) \times\left(1-\operatorname{Assign}\left(\operatorname{Src}, \text { Des }, C_{i}\right)\right) \\
& \times U D G(\operatorname{Src}, \text { Des }) \times \text { Link_(Src,Des }) \\
& \text { Src, Des, temp } 1, \text { temp } 2 \in \text { Nodes, } C_{i} \in \text { ChannelList. }
\end{aligned}
$$

And finally, the objective of the CA_Model is:

\section{MinimiseTotal_Interference}

Therefore, the BIP model for channel assignment subproblem of the layered scheme is represented as follows:

\section{Minimise Total_Interference}

$$
\begin{aligned}
& \text { Subject to: } \\
& \sum_{C_{i}} \operatorname{Assign}\left(\operatorname{Src}, \operatorname{Des}, C_{i}\right)=\operatorname{Link}(\operatorname{Src}, \text { Des }) \text {, } \\
& \text { Src, Des } \in \text { Nodes, } C_{i} \in \text { ChannelList } \\
& U D G(\operatorname{Src}, \text { Des }) \times \text { Link_(Src,Des }) \\
& \times \text { Interference }\left(\operatorname{Src}, \text { Des, } C_{i}\right) \\
& \leq\left(\left(\sum_{\text {temp } 1} U D G(\operatorname{Src}, \text { temp } 1) \times\left(\sum_{\text {temp } 1} U D G(\operatorname{Src}, \text { temp } 1)-1\right)\right)\right. \\
& \left.+\left(\sum_{\text {temp } 2} U D G(\text { Des, temp } 2) \times\left(\sum_{\text {temp } 2} U D G(\text { Des, temp } 2)-1\right)\right)\right) \\
& \times \operatorname{Assign}\left(\operatorname{Src}, \operatorname{Des}, C_{i}\right) \times U D G(\operatorname{Src}, \text { Des }) \\
& \times \text { Link_(Src,Des) }
\end{aligned}
$$

$$
\begin{aligned}
& \text { UDG }(\text { Src }, \text { Des }) \times \text { Link_(Src,Des }) \\
& \times \operatorname{Interference}\left(\operatorname{Src}, \text { Des }, C_{i}\right) \leq \operatorname{SIL}\left(\operatorname{Src}, \text { Des }, C_{i}\right) \\
& \times U D G(\operatorname{Src}, \text { Des }) \times \text { Link_(Src,Des }) \\
& \text { Src }, \text { Des } \in \text { Nodes }, C_{i} \in \text { ChannelList }
\end{aligned}
$$

$U D G(\operatorname{Src}$, Des $) \times$ Link_(Src,Des $)$

$\times$ Interference $\left(\operatorname{Src}\right.$, Des,$\left.C_{i}\right) \geq\left(\operatorname{SIL}\left(\operatorname{Src}, \operatorname{Des}, C_{i}\right)\right.$

$-\left(\sum_{\text {temp } 1} U D G(\operatorname{Src}\right.$, temp 1$) \times\left(\sum_{\text {temp } 1} U D G(\operatorname{Src}\right.$, temp 1$\left.\left.)-1\right)\right)$

$+\left(\sum_{\text {temp } 2} U D G(\right.$ Des, temp 2$) \times\left(\sum_{\text {temp } 2} U D G(\right.$ Des, temp 2$\left.\left.\left.)-1\right)\right)\right)$

$\times\left(1-\operatorname{Assign}\left(\operatorname{Src}, \operatorname{Des}, C_{i}\right)\right) \times U D G(\operatorname{Src}, D e s)$

$\times$ Link_(Src,Des),

Src, Des, temp 1, temp $2 \in$ Nodes,$C_{i} \in$ ChannelList

\section{Connectivity and loop considerations}

We tend to demonstrate the immunity of our formulation to loop formations as well as to ensure connectivity that is preserved with the resulting channel-radio assignments. Our essential reason for the worst case is also evidently applicable to the average scenario.

\subsection{Connectivity}

We examine the multicast tree connectivity in the proposed cross-layer method. A similar line of reasoning is also applicable to the case of our Sequential design.

Definition 1. Connectivity is satisfied only if there exists a path between the multicast source and all multicast group members.

Property 1. The BIP formulation given in Section 3 guarantees connectivity across the multicast tree.

Proof: We demonstrate the notion of connectivity using the Unit Disk Graph (UDG) depicted in Figure 2(a) without loss of generality. In this graph, each link between two nodes indicates that they are located within the transmission range of each other. Two inter-linked nodes would be able to communicate if only identical channel numbers get assigned to one of their radios. MS denotes the multicast source and MT stands for the multicast target.

Constraint (11) ensures that MT has exactly one incoming link, as illustrated in Figure 2(b). Constraints (14) and (15) stipulate that in case a mesh node has an outgoing link, it ought to have an incoming link as well. Inequality (6) requires that only one of the links $(1,2)$ or $(3,2)$ be established as demonstrated in Figure 2(c). On the other hand, constraint (10) warrants that MS has at least one outgoing link. Therefore, mesh node 1 has to be necessarily associated with an output and the outgoing link from node 3 should be removed (Fig. 2(d)). Hence, there exists a path from MS to MT.

Figure 2 Multicast tree connectivity

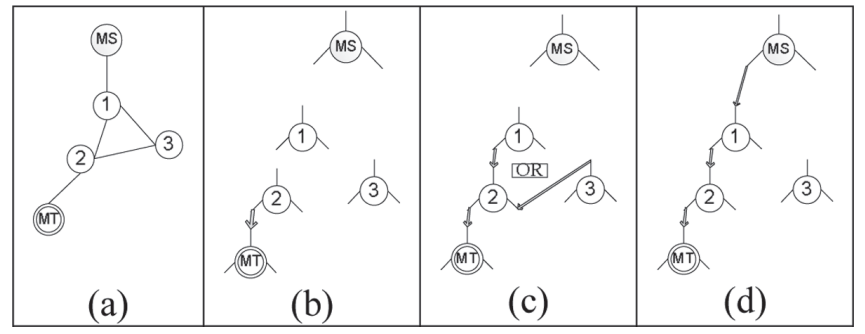

\subsection{Loop occurrence}

We investigate the correctness of our proposed formulation for the proposed cross-layer method in terms of the loop formation issue through the following property. Here again we have considered the worst case scenario. A similar analysis applies to the Sequential scheme.

Property 2. The multicast tree associated with the BIP formulation given in Section 3 is guaranteed to be loopfree. 
Proof: Given the UDG depicted in Figure 2(a), we assume that there exists a loop, say $(1,2,3,1)$ within the multicast tree, as shown in Figure 3(a). Constraints (9) and (10) ensure that MS has at least one outgoing link, as shown in Figure 3(b). Inequality (6) requires that link $(3,1)$ be removed from the configuration (Fig. 3(c)). Also, constraints (14) and (15) warrant that node 3 has no incoming link (Fig. 3(d)). Finally, Constraint (11) stipulates that the MT has exactly one incoming link (Fig. 3(e)).

Figure 3 Multicast tree loop prevention

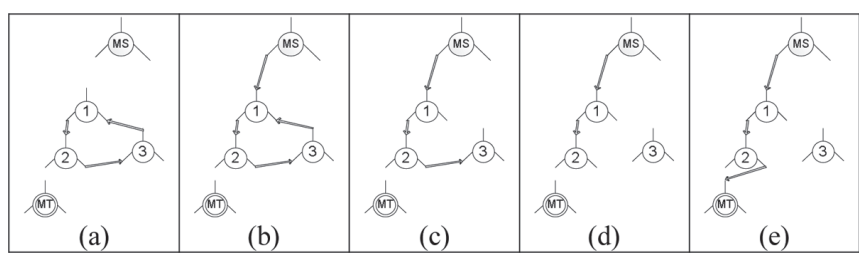

\section{Simulation results}

We implemented our BIP framework using CPLEX 11 (for more information about CPLEX, see Ilog Inc. (2011)). Further, to evaluate the performance of the proposed methods, several simulations have been conducted. Simulation 1 was conducted to observe the impact of channel number variations on the overall interference in our cross-layer design, as compared with LCA, MCM (Zeng et al., 2007, 2010) and methods based on the GA, SA and the Tabu search (Cheng and Yang, 2008a, 2008b, 2011). In simulation 2, the proposed cross-layer and Sequential methods are evaluated in terms of optimality, say overall interference, on the one hand and complexity, say required time to solve the multicast problem and memory consumption, on the other hand. Our Sequential method is compared with methods based on LCA, MCM, GA, SA and TS in simulation 3. Simulation 4 is dedicated to compare the resultant interference for different methods considering radio number variations. All simulations were conducted on a dense random generated UDG in which the number of nodes and the size of multicast receivers set are 30 and 13 respectively. Simulations are described as follows.

\subsection{Simulation 1}

In this simulation, we study the resultant interference in the proposed cross-layer design as compared with LCA and MCM, as well as with methods based on GA, SA and TS. Recall from section 1 that the number of channels in MCMR WMNs should be more than or equal to the number of radios. Therefore, for a network with $n$-radio nodes, a set of simulations for $n$ through $m$ channels should be conducted ( $m \geq n)$, where $m$ is the point at which interference reaches zero. Figures 4(a) through 4(f) show the resultant interference for different methods, when the mesh nodes are equipped with different numbers of radios. In Figures 4(a)-(e), every curve is the result of a simulation set, not just one simulation. In other words, every data point shows the result of a simulation in a simulation set. Each data point associated with methods based on LCA, MCM, GA, SA and $\mathrm{TS}$ in this simulation is the average of 20 times simulations. Since utilising the methods based on GA, SA and TS in 3- and 4-radio networks leads to disconnection of the wireless mesh network, their corresponding results have not been shown in Figures 4(a) and (b).

Figure 4 Impact of channel number variations on the overall interference (see online version for colours)
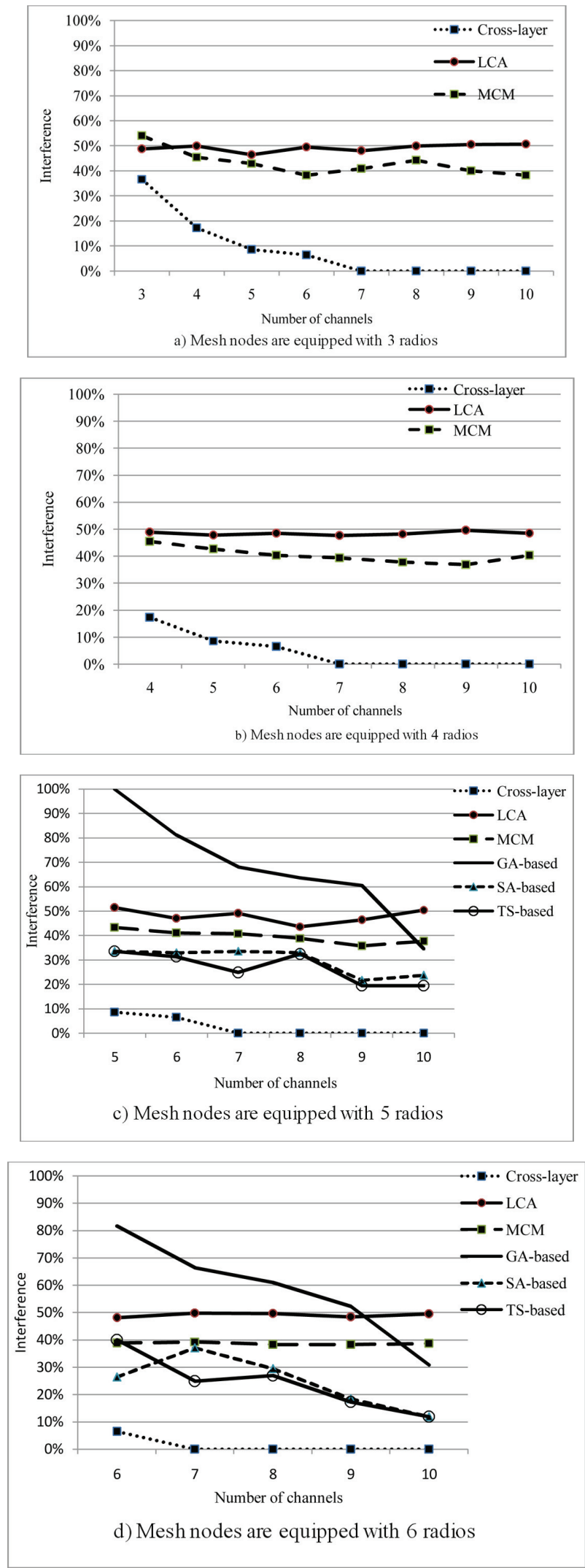
Figure 4 Impact of channel number variations on the overall interference (see online version for colours) (continued)

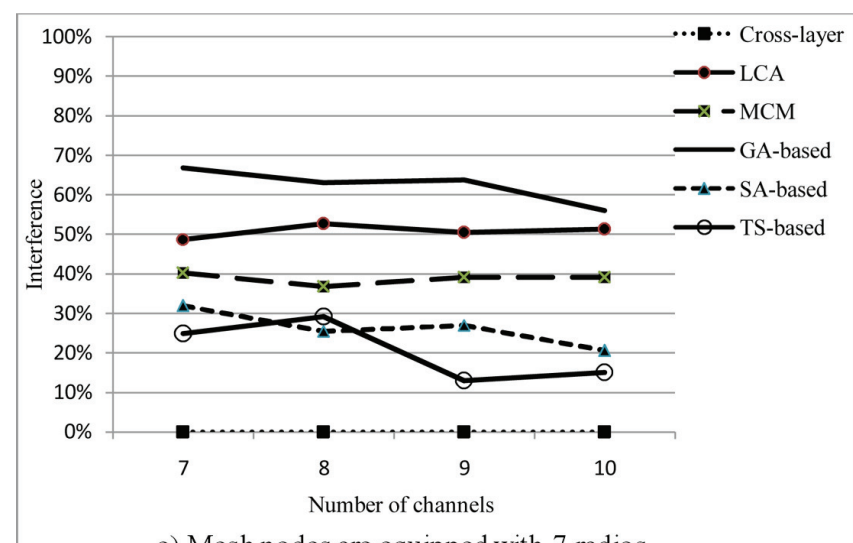

e) Mesh nodes are equipped with 7 radios

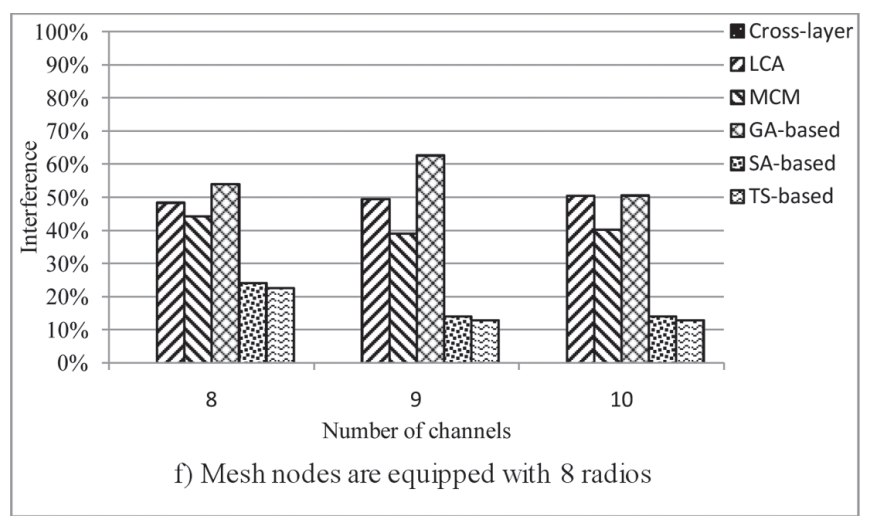

The results demonstrate that in a network with the same number of radios, increasing the number of channels leads to interference reduction in the proposed cross-layer design. From the results shown in all charts of Figure 4, as is expected, our method achieves minimal interference. The reason is that our cross-layer design is based on the BIP formulation, in which two sub-problems are solved conjointly. In other words, the proposed cross-layer design considers the impact of the routing and MAC layers on each other and hence, an optimal solution for the overall problem is provided. From the results, the overall interference from running LCA and MCM has no specific trend. The reason is that in these algorithms there is no channel/radio number considerations during the multicast tree construction and channel assignment phases. The results obtained from this simulation show that the TS-based method outperforms other heuristic- and meta-heuristic-based methods in terms of interference. The reason is that using the Tabu search technique will result in escape from the local optima and intelligent search of the whole solution space. Figure 4(f) shows that by applying the cross-layer design in a MCMR WMN with 8-radio nodes, with 8, 9 and 10 available channels, interference becomes zero. Other bar charts in the following simulations can be interpreted analogously.

\subsection{Simulation 2}

This simulation is conducted to compare the optimality of our proposed cross-layer and Sequential designs in terms of overall interference. The simulations' configuration is the same as simulation 1. As illustrated by Figure 5, the cross-layer method marginally outperforms the Sequential method in terms of interference in all network configurations. The reason is that solving both sub-problems sequentially and achieving the optimal solution for each one does not guarantee the optimal solution of the overall problem.

Figure 5 Impact of channel number variations on interference for the proposed cross-layer and Sequential methods
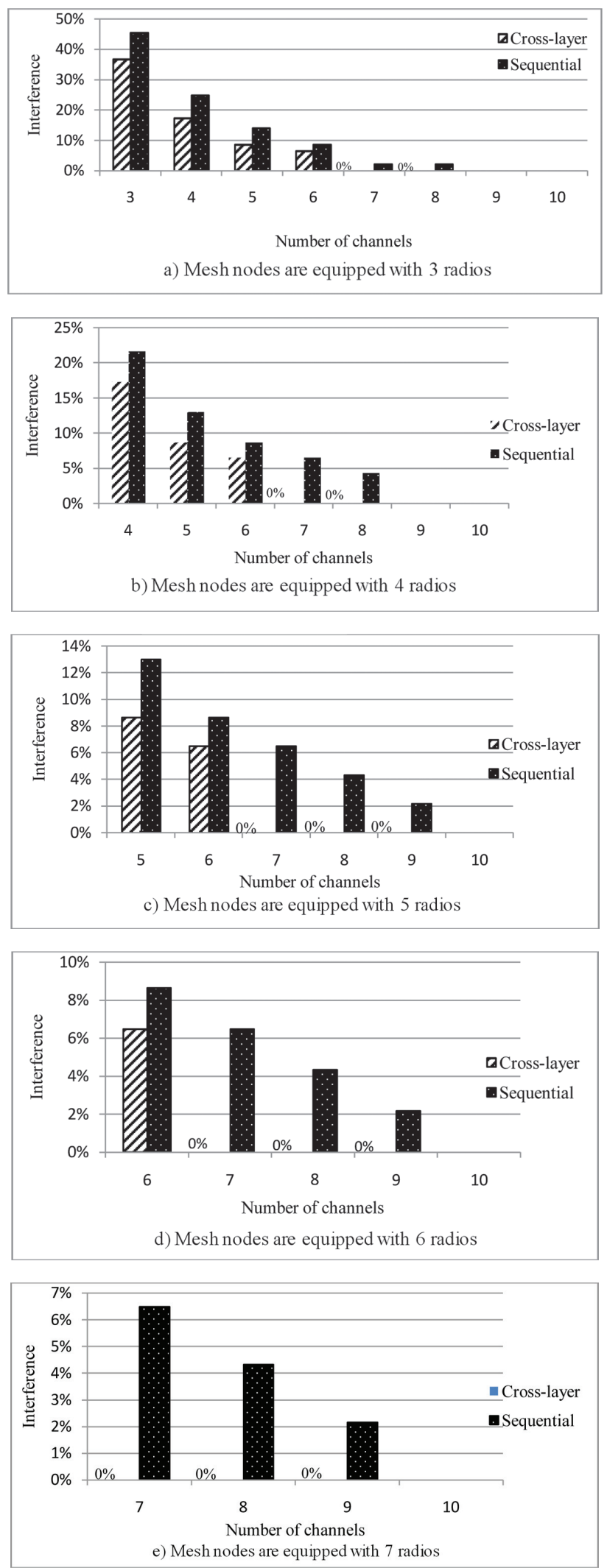
Figure 5 Impact of channel number variations on interference for the proposed cross-layer and Sequential methods (continued)
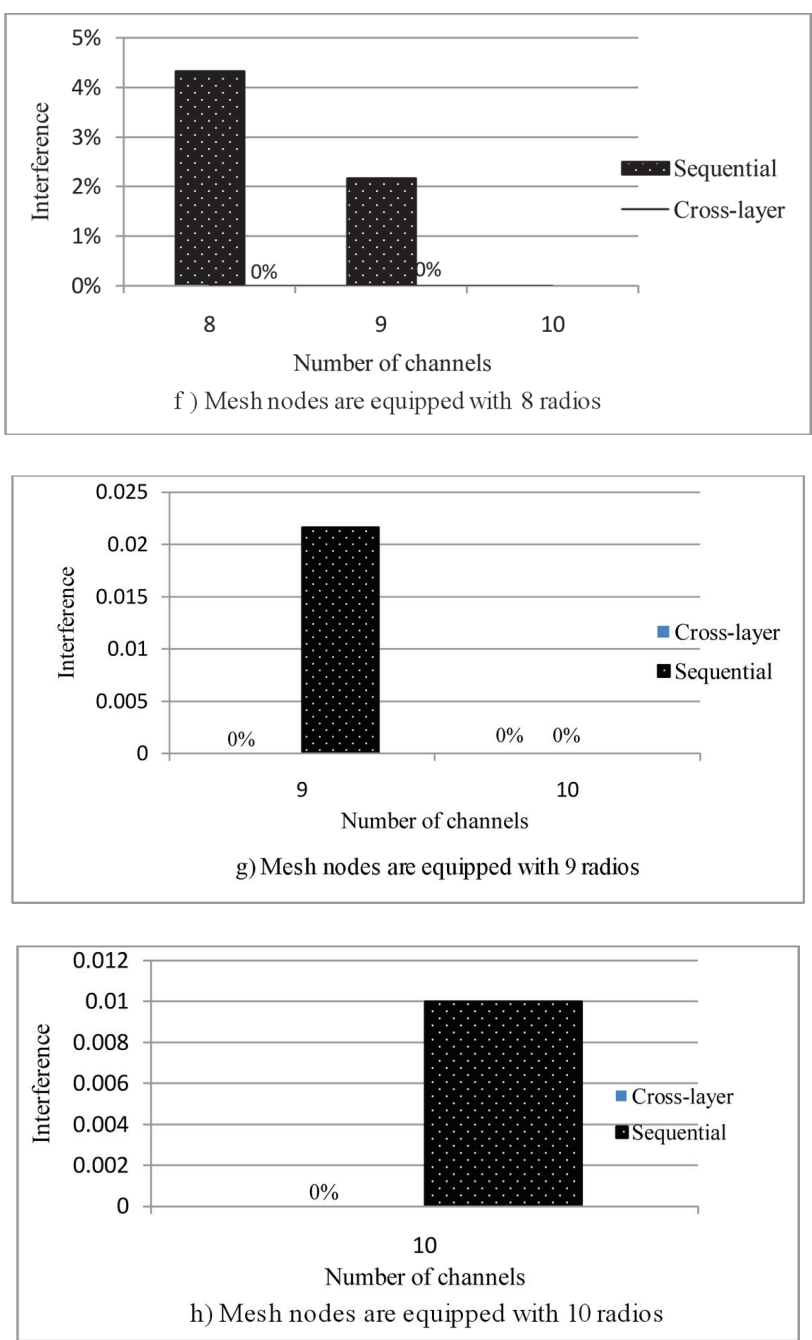

In this simulation, we compared the complexity of the two proposed methods as well. Table 4 shows the percentage of the constraints in both proposed methods. The more the number of constraints in a given formulation is, the higher the number of comparison operations and hence, the longer it takes to solve the problem. As can be seen, in all network configurations, the constraints percentage in the Sequential design is less than that of the cross-layer design, resulting in smaller multicast problem solving time.

In this simulation, we also recorded the number of required variables to solve the problem. More variables lead to more memory demands to solve the problem. The results are in Figure 6, in which the legend $\mathrm{CnS}$ denotes that the corresponding curve is the result of a simulation set obtained from running the proposed Sequential method where the number of channels is fixed at $n$. Further, legends $C n C L$ are associated with the results of a simulation set obtained from running the proposed cross-layer design. Our observation is that the number of variables in the Sequential method as compared with the cross-layer method is dramatically decreased. Besides, memory management in the cross-layer method makes the processor busy and hence, again leads to higher solving time.
Table 4 percentage of the constraints in both proposed methods

\begin{tabular}{|c|c|c|c|}
\hline \multirow[b]{2}{*}{ Ch\# } & \multirow[b]{2}{*}{$R \#$} & \multicolumn{2}{|c|}{ Number of constraints } \\
\hline & & Sequential method\% & Cross-layer method\% \\
\hline 3 & 3 & 3.937 & 3.992 \\
\hline \multirow[t]{2}{*}{4} & 3 & 5.181 & 5.302 \\
\hline & 4 & 8.991 & 9.122 \\
\hline \multirow[t]{3}{*}{5} & 3 & 6.421 & 6.613 \\
\hline & 4 & 11.183 & 11.387 \\
\hline & 5 & 17.320 & 17.523 \\
\hline \multirow[t]{4}{*}{6} & 3 & 7.665 & 7.923 \\
\hline & 4 & 13.382 & 13.651 \\
\hline & 5 & 20.741 & 20.893 \\
\hline & 6 & 29.735 & 30.012 \\
\hline \multirow[t]{5}{*}{7} & 3 & 8.907 & 9.234 \\
\hline & 4 & 15.577 & 15.916 \\
\hline & 5 & 24.162 & 24.358 \\
\hline & 6 & 34.635 & 34.945 \\
\hline & 7 & 47.059 & 47.260 \\
\hline \multirow[t]{6}{*}{8} & 3 & 10.150 & 10.545 \\
\hline & 4 & 17.772 & 18.012 \\
\hline & 5 & 27.583 & 27.827 \\
\hline & 6 & 39.574 & 39.884 \\
\hline & 7 & 53.749 & 54.033 \\
\hline & 8 & 70.106 & 70.391 \\
\hline \multirow[t]{7}{*}{9} & 3 & 11.393 & 11.832 \\
\hline & 4 & 19.967 & 20.255 \\
\hline & 5 & 31.004 & 31.424 \\
\hline & 6 & 44.494 & 44.908 \\
\hline & 7 & 60.440 & 60.787 \\
\hline & 8 & 78.841 & 79.141 \\
\hline & 9 & 99.694 & 100 \\
\hline
\end{tabular}

Figure 6 percentage of the memory demands in both cross-layer and Sequential methods

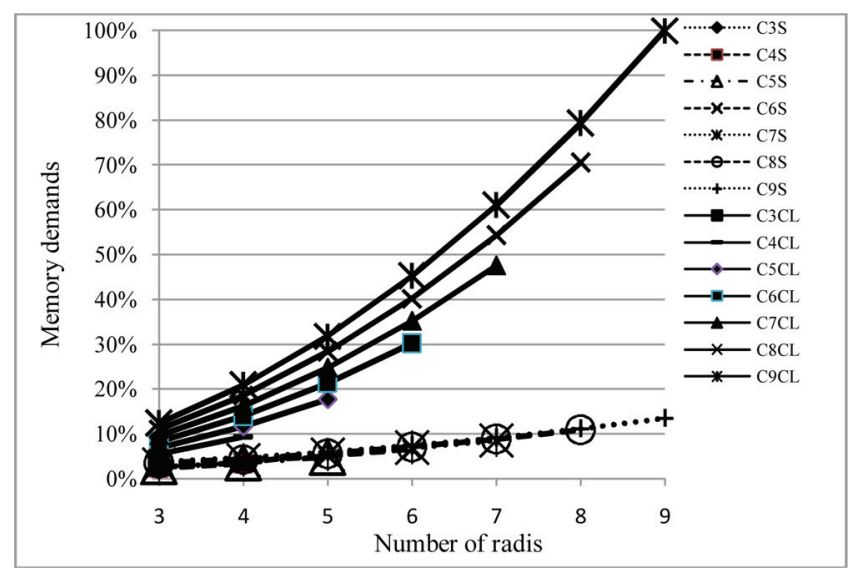

\subsection{Simulation 3}

This simulation is the same as simulation 1 and was conducted to evaluate the resultant interference of our proposed Sequential design as compared with the methods based on LCA, MCM, GA, SA and TS. The results are in Figure 7. As mentioned in simulation 1, applying methods based on GA, SA and TS leads the network to a situation where 3 and 4 radios per each node need to be disconnected and hence, their associated curves are not shown in Figures 7(a) and 
Figure 7 Impact of channel number variations on interference for different method (see online version for colours)
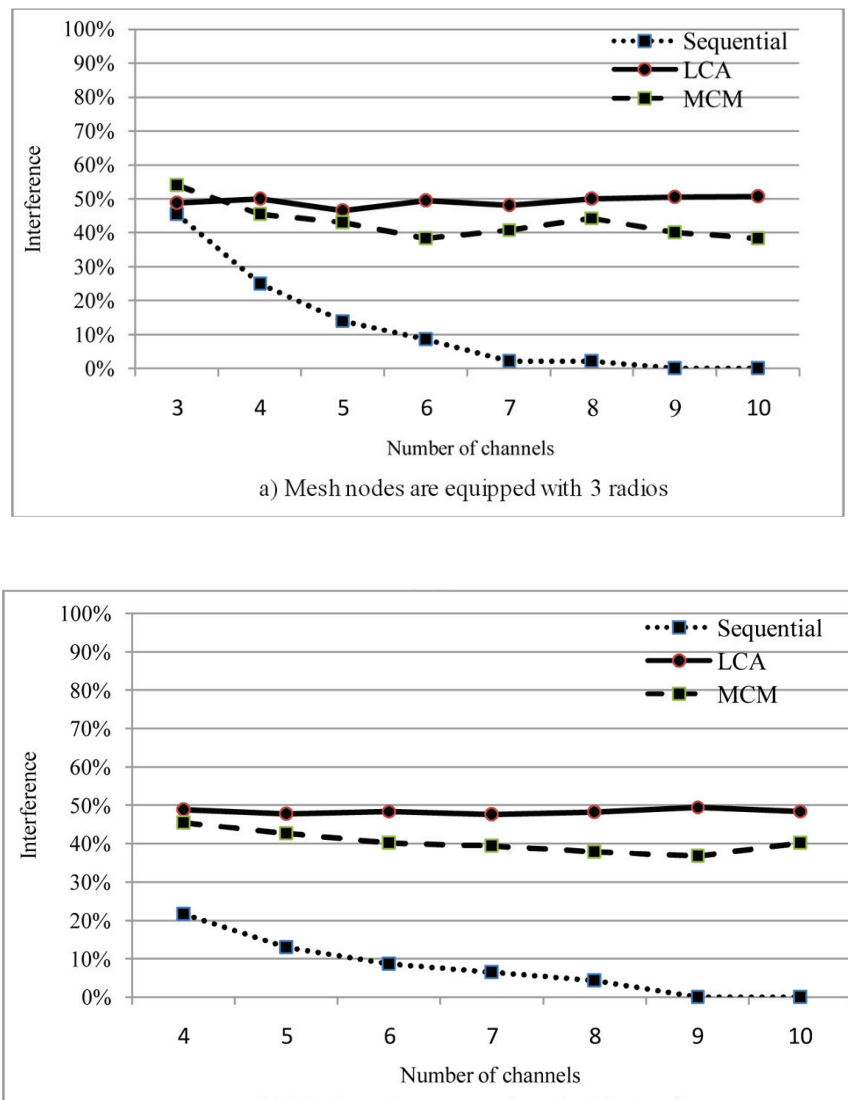

b) Mesh nodes are equipped with 4 radios

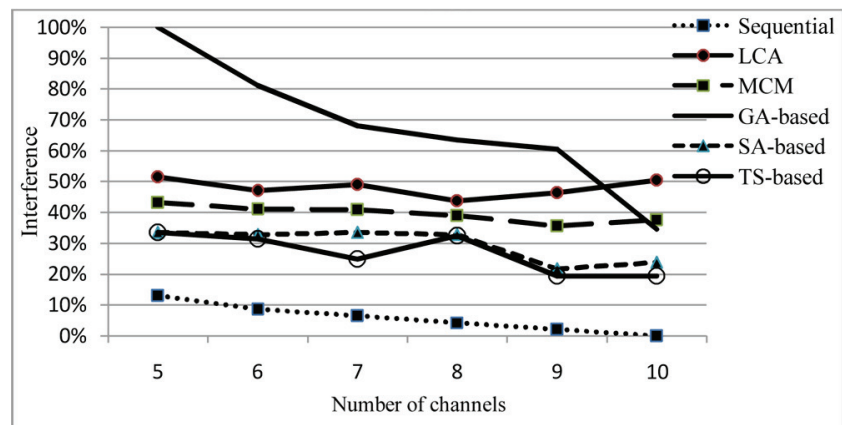

c) Mesh nodes are equipped with 5 radios

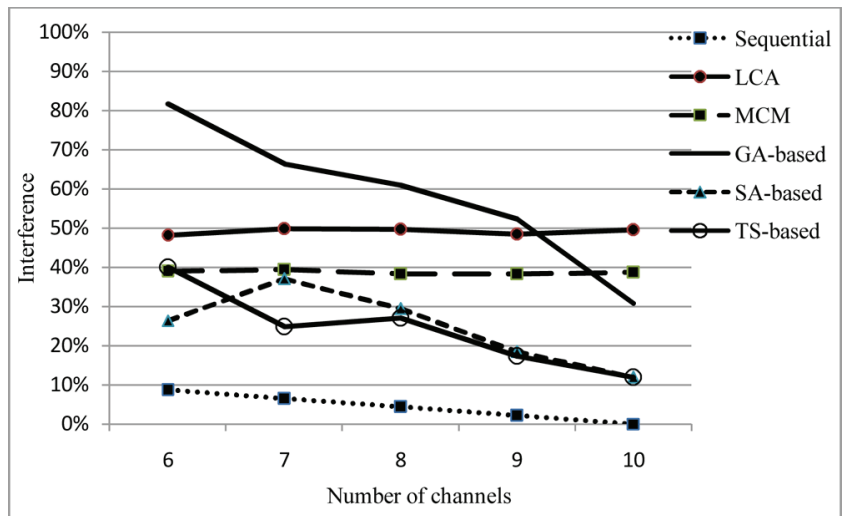

d) Mesh nodes are equipped with 6 radios
Figure 7 Impact of channel number variations on interference for different method (see online version for colours) (continued)
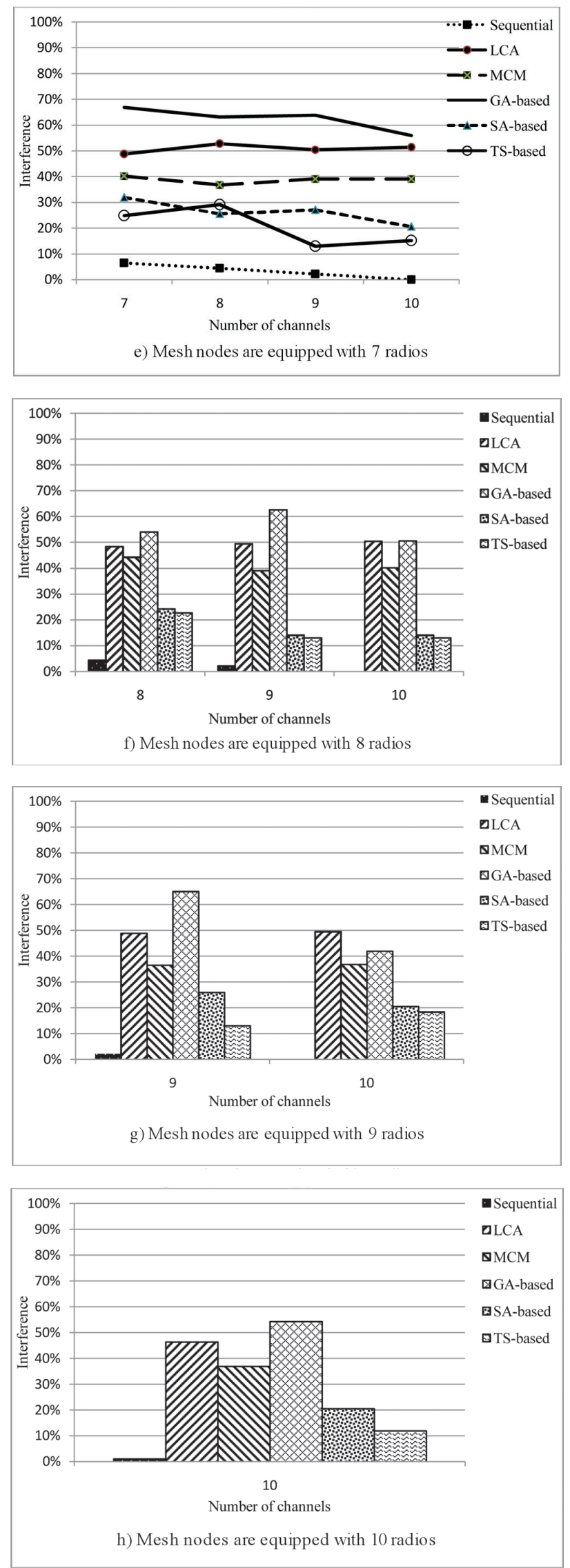
(b). The results demonstrate that in a network with the same number of radios, increasing the number of channels leads to interference reduction in the proposed Sequential method. As is seen in this figure, the proposed Sequential method outperforms other methods in terms of interference.

\subsection{Simulation 4}

Unlike simulations 1 through 3 , this simulation was conducted to study the impact of radio number variations on the resultant interference for different methods. Recall from Section 1 that the number of radios is not more than the number of channels. Therefore, in a network with $n$ available channels, every node can have 2 to $n$ radios. In all methods, using two radios leads to the given network being disconnected. Hence, the number of radios in the simulations was considered from 3 to the number of channels. The percentages of interference obtained from applying different methods are summarised in Table 5. The columns $C h \#$ and $R \#$ denote the number of channels and radios, respectively. Utilising methods based on GA, SA and TS in 3- and 4-radio networks leads to the network being disconnected. These situations are shown with ' $D C$ ' in Table 5. We ran the algorithms based on LCA, MCM, GA, SA and TS 20 times and averaged the results. This table demonstrates that the sequential design with marginally more interference as compared with the cross-layer design stands in the second place of optimality.

Table 5 Percentage of obtained interference in different methods regarding radio number variations

\begin{tabular}{|c|c|c|c|c|c|c|c|c|}
\hline Ch\# & $R \#$ & $L C A$ & $M C M$ & $\begin{array}{l}\text { GA-based } \\
\text { method }\end{array}$ & $\begin{array}{l}\text { SA-based } \\
\text { method }\end{array}$ & $\begin{array}{l}\text { TS-based } \\
\text { method }\end{array}$ & $\begin{array}{c}\text { Proposed sequential } \\
\text { method }\end{array}$ & $\begin{array}{l}\text { Proposed cross- } \\
\text { layer method }\end{array}$ \\
\hline 3 & 3 & 48.75676 & 54.05405 & $\mathrm{DC}$ & $\mathrm{DC}$ & DC & 45.40541 & 36.75676 \\
\hline \multirow[t]{2}{*}{4} & 3 & 49.94595 & 45.40541 & $\mathrm{DC}$ & $\mathrm{DC}$ & $\mathrm{DC}$ & 24.86486 & 17.2973 \\
\hline & 4 & 48.86486 & 45.40541 & $\mathrm{DC}$ & $\mathrm{DC}$ & DC & 21.62162 & 17.2973 \\
\hline \multirow[t]{3}{*}{5} & 3 & 46.48649 & 43.02703 & $\mathrm{DC}$ & $\mathrm{DC}$ & $\mathrm{DC}$ & 14.05405 & 8.648649 \\
\hline & 4 & 47.78378 & 42.59459 & $\mathrm{DC}$ & $\mathrm{DC}$ & $\mathrm{DC}$ & 12.97297 & 8.648649 \\
\hline & 5 & 51.45946 & 43.24324 & 100 & 33.51351 & 33.5135 & 12.97297 & 8.648649 \\
\hline \multirow[t]{4}{*}{6} & 3 & 49.51351 & 38.27027 & $\mathrm{DC}$ & $\mathrm{DC}$ & DC & 8.648649 & 6.486486 \\
\hline & 4 & 48.43243 & 40.21622 & $\mathrm{DC}$ & $\mathrm{DC}$ & $\mathrm{DC}$ & 12.97297 & 6.486486 \\
\hline & 5 & 47.02703 & 41.08108 & 81.08108 & 32.86486 & 31.3514 & 8.648649 & 6.486486 \\
\hline & 6 & 48.10811 & 38.91892 & 81.62162 & 26.37838 & 40 & 8.648649 & 6.486486 \\
\hline \multirow[t]{5}{*}{7} & 3 & 48.10811 & 40.86486 & $\mathrm{DC}$ & $\mathrm{DC}$ & $\mathrm{DC}$ & 2.162162 & 0 \\
\hline & 4 & 47.56757 & 39.35135 & $\mathrm{DC}$ & $\mathrm{DC}$ & $\mathrm{DC}$ & 6.486486 & 0 \\
\hline & 5 & 49.08108 & 40.86486 & 68.10811 & 33.51351 & 24.8649 & 6.486486 & 0 \\
\hline & 6 & 49.83784 & 39.35135 & 66.37838 & 37.05946 & 24.8649 & 6.486486 & 0 \\
\hline & 7 & 48.75676 & 40.21622 & 66.81081 & 31.89189 & 24.8649 & 6.486486 & 0 \\
\hline \multirow[t]{6}{*}{8} & 3 & 49.94595 & 44.32432 & $\mathrm{DC}$ & $\mathrm{DC}$ & $\mathrm{DC}$ & 2.162162 & 0 \\
\hline & 4 & 48.21622 & 37.83784 & $\mathrm{DC}$ & $\mathrm{DC}$ & DC & 4.324324 & 0 \\
\hline & 5 & 43.67568 & 38.91892 & 63.56757 & 32.86486 & 32.4324 & 4.324324 & 0 \\
\hline & 6 & 49.62162 & 38.27027 & 60.97297 & 29.40541 & 27.027 & 4.324324 & 0 \\
\hline & 7 & 52.75676 & 36.75676 & 63.13514 & 25.51351 & 29.1892 & 4.324324 & 0 \\
\hline & 8 & 48.43243 & 44.32432 & 54.05405 & 24.21622 & 22.7027 & 4.324324 & 0 \\
\hline \multirow[t]{7}{*}{9} & 3 & 50.48649 & 40 & $\mathrm{DC}$ & $\mathrm{DC}$ & $\mathrm{DC}$ & 0 & 0 \\
\hline & 4 & 49.51351 & 36.75676 & $\mathrm{DC}$ & $\mathrm{DC}$ & $\mathrm{DC}$ & 0 & 0 \\
\hline & 5 & 46.37838 & 35.67568 & 60.54054 & 21.62162 & 19.4595 & 2.162162 & 0 \\
\hline & 6 & 48.43243 & 38.27027 & 52.32432 & 18.37838 & 17.2973 & 2.162162 & 0 \\
\hline & 7 & 50.48649 & 39.13514 & 63.78378 & 27.02703 & 12.973 & 2.162162 & 0 \\
\hline & 8 & 49.51351 & 39.13514 & 62.7027 & 14.05405 & 12.973 & 2.162162 & 0 \\
\hline & 9 & 48.86486 & 36.54054 & 65.08108 & 25.94595 & 12.973 & 2.162162 & 0 \\
\hline \multirow[t]{8}{*}{10} & 3 & 50.7027 & 38.27027 & $\mathrm{DC}$ & $\mathrm{DC}$ & $\mathrm{DC}$ & 0 & 0 \\
\hline & 4 & 48.43243 & 40.21622 & $\mathrm{DC}$ & $\mathrm{DC}$ & $\mathrm{DC}$ & 0 & 0 \\
\hline & 5 & 50.48649 & 37.62162 & 34.59459 & 23.78378 & 19.4595 & 0 & 0 \\
\hline & 6 & 49.51351 & 38.7027 & 30.81081 & 11.89189 & 11.8919 & 0 & 0 \\
\hline & 7 & 51.35135 & 39.13514 & 56 & 20.54054 & 15.1351 & 0 & 0 \\
\hline & 8 & 50.48649 & 40.21622 & 50.59459 & 14.05405 & 12.973 & 0 & 0 \\
\hline & 9 & 49.51351 & 36.75676 & 41.94595 & 20.54054 & 18.3784 & 0 & 0 \\
\hline & 10 & 46.37838 & 36.97297 & 54.27027 & 20.54054 & 11.8919 & 0 & 0 \\
\hline
\end{tabular}


From the results, the obtained interference in the Sequential method with 8 channels and 3 radios is around 2\%. But by increasing the number of radios to 4 or more leads, the interference comes to almost $4.32 \%$. The reason is that if the number of radios is increased, then the Sequential design has more chance to find the optimal MCT multicast tree. In this case, the optimal solution for the multicast tree construction is provided, but the resultant tree may not be a suitable input to the channel assignment phase such that the overall problem solution is optimised. This is the weakness of all sequential methods.

It is also observed that increasing the number of radios in the Sequential method will be useful to a point. For example, in a network with 5 available channels, if the number of radios varies from 3 to 4 , a multicast tree with interference of almost 12.97 is constructed. But as can be observed, increasing the number of radios no longer has an impact on the resulting multicast tree and interference reduction. This threshold is dependent on the network topology.

In sum, according to the conducted simulations, the resultant interference is primarily driven by the number of channels rather than the number of radios. Extensive simulation results demonstrate that the overall interference computed by the proposed methods as compared with the methods based on LCA, MCM, GA, SA and TS is comprehensively low and hence, outperforms them. Among the two proposed approaches, the cross-layer method gives the optimal solution for the joint channel assignment and the multicast tree construction problem. However, the cross-layer design benefits always come together with difficulties. This means that by increasing the size of the problem, the cross-layer method loses its efficiency due to more computational complexity and memory demands. In this case, the proposed Sequential method solves the problem in an efficient way.

\section{Performance analysis}

In this section, the efficiency of two proposed designs regarding two metrics' memory demands and time complexity are investigated analytically.

\subsection{Memory demands}

During implementation, memory requirements for each method can be defined as a function of the generated variables. In fact, the number of generated variables for each corresponding variable defined within the model depends on the number of different combinations of the variable's input set. For example, the number of generated variables for the modelled variable link(Node, Radios, Node, Radio, Channel) can be computed as $\mid$ Node $\left.\right|^{2} \times \mid$ Radios $\left.\right|^{2} \times \mid$ ChannelList $\mid$. Therefore, the number of all Integers and Binary variables for the cross-layer design is computed as equations (45) and (46), respectively.

$$
2 \times\left(\mid \text { Node }\left.\right|^{2}+\mid \text { Node }|+| \text { Radios }\left.\right|^{2}+\mid \text { ChannelList } \mid+1\right)
$$

$$
\begin{gathered}
\mid \text { Node }\left.\right|^{2} \times \mid \text { Radios }\left.\right|^{2} \times \mid \text { ChannelList } \mid \\
+\mid \text { Node }|\times| \text { Radios }|\times| \text { ChannelList } \mid
\end{gathered}
$$

Table 6 also shows the number of all Integer and Binary variables for each sub-problem of the proposed Sequential design.

Table 6 The number of integer/binary variables within Sequential design

\begin{tabular}{lll}
\hline $\begin{array}{l}\text { Sub- } \\
\text { problem }\end{array}$ & $\begin{array}{l}\text { Number of binary } \\
\text { variables }\end{array}$ & Number of integer variables \\
\hline $\begin{array}{l}\text { Multicast } \\
\text { tree }\end{array}$ & $\mid$ Node $\left.\right|^{2} \times \mid$ Radios $\left.\right|^{2}$ & $2 \times \mid$ Node $\mid+1$ \\
$\begin{array}{l}\text { construction } \\
\begin{array}{l}\text { Channel } \\
\text { assignment }\end{array}\end{array}$ & $\mid$ Node $\left.\right|^{2} \times \mid$ ChannelList $\mid$ & $2 \times \mid$ Node $\left.\right|^{2} \times \mid$ ChannelList $\mid+1$ \\
\hline
\end{tabular}

From the above analysis, it can be concluded that the number of binary variables in the cross-layer design is larger than the sum of the binary variables of both sub-problems in the Sequential design. Similarly, the number of integer variables in the cross-layer design is larger than the sum of the integer variables specified in both sub-problems of the Sequential method. That is, the number of all generated variables in the cross-layer design is more than that of the layered design, which leads to more memory demands. Besides, due to the existence of a larger solution space in the cross-layer design, it is expected that solving the cross-layer formulation as compared with the Sequential design needs more time.

\subsection{Time complexity}

Every linear integer programming problem with bounded variables can be converted into a linear binary programming problem (Taha, 1975). That is, our problem can be converted to strict binary programming. Besides, the coefficient matrix of our network-based formulation is totally uni-modular (Bazaraa et al., 2011). This feature helps us to model continues variables in the form of $x_{i j} \geq 0$ instead of binary variables. In this case, it is assured that the optimal solution of the new problem is binary and it can be solved in $O\left(m^{2} n\right)$, in which $m$ and $n$ are the number of constraints and variables, respectively (Bazaraa et al., 2011).

To analyse the time complexity of the proposed cross-layer design, we initially compute the number of constraints of this model. Table 7 lists the number of generated constraints for each corresponding constraint within the cross-layer model. In summary, the number of constraints in the cross-layer design is $8 \mid$ Nodes $\left.\right|^{2} \mid$ Radios $\left.\right|^{2} \mid$ ChannelList $|+4|$ Nodes $\mid+1$.

In what follows, the number of binary variables within the cross-layer model is computed; Every integer variable with a maximum value of $k$ can be modelled with $\log (k)+1$ binary variables. Therefore, we can model the integer variables of the cross-layer design with binary variables. For example, equation (1) is defined for each node of networks. The value of each one is at most $(\mid$ Nodes $\mid-1)$, which can be modelled with $[\log (\mid$ Nodes $\mid-1)]+1$ binary variables. In other words, the maximum number of binary variables for equation (1) 
is defined as $\mid$ Nodes $\mid \log (\mid$ Nodes $\mid-1)$. Therefore, the whole number of converted binary variables within the cross-layer design is:

$$
\begin{aligned}
& \frac{1}{2}\left(\mid \text { Nodes }||^{4} \mid \text { Radios }\left.\right|^{2} \mid \text { ChannelList } \mid\right. \\
& \left.-\mid \text { Nodes }\left.\right|^{3} \mid \text { Radios }\left.\right|^{6} \mid \text { ChannelList } \mid\right) \\
& +\mid \text { Nodes } \mid(\mid \text { Nodes } \mid-1)+2 \mid \text { Nodes } \mid[\log (\mid \text { Nodes } \mid-1)] \text {. }
\end{aligned}
$$

Table 7 The number of generated constraints for each constraint of formulation

\begin{tabular}{ll}
\hline $\begin{array}{l}\text { Constraint in } \\
\text { cross-layer model }\end{array}$ & $\begin{array}{l}\text { Number of constraints during } \\
\text { implementation }\end{array}$ \\
\hline
\end{tabular}

\begin{tabular}{ll}
\hline$(6)$ & $\mid$ Nodes $|-|$ MulticastTarget $\mid-1$ \\
$(7)$ & $\mid$ Nodes $|-|$ MulticastTarget $\mid-1$ \\
$(8)$ & 1 \\
$(9)$ & 1 \\
$(10)$ & 1 \\
$(11)$ & $\mid$ MulticastTarget $\mid$ \\
$(12)$ & $\mid$ MulticastTarget $\mid$ \\
$(13)$ & $\mid$ Nodes $\left.\right|^{2} \times \mid$ Radios $\left.\right|^{2} \times \mid$ ChannelList $\mid$ \\
$(14)$ & $\mid$ Nodes $\mid$ \\
$(15)$ & $\mid$ Nodes $\mid$ \\
$(16)$ & $\mid$ Nodes $\left.\right|^{2} \times \mid$ Radios $\left.\right|^{2} \times \mid$ ChannelList $\mid$ \\
$(17)$ & $\mid$ Nodes $\left.\right|^{2} \times \mid$ Radios $\left.\right|^{2} \times \mid$ ChannelList $\mid$ \\
$(18)$ & $\mid$ Nodes $\left.\right|^{2} \times \mid$ Radios $\left.\right|^{2} \times \mid$ ChannelList $\mid$ \\
$(19)$ & $\mid$ Nodes $\left.\right|^{2} \times \mid$ Radios $\left.\right|^{2} \times \mid$ ChannelList $\mid$ \\
$(20)$ & $\mid$ Nodes $\left.\right|^{2} \times \mid$ Radios $\left.\right|^{2} \times \mid$ ChannelList $\mid$ \\
$(21)$ & $\mid$ Nodes $\left.\right|^{2} \times \mid$ Radios $\left.\right|^{2} \times \mid$ ChannelList $\mid$ \\
\hline &
\end{tabular}

We also had the number of $\left(\mid\right.$ Nodes $\left.\right|^{2} \mid$ Radios $\left.\right|^{2} \mid$ ChannelList $\mid$ $+\mid$ Nodes $\|$ Radios || ChannelList $\mid)$ binary variables in the original model. From the above, the time complexity of the cross-layer design is $O\left(\mid\right.$ Nodes $\left.\right|^{8} \mid$ Radios $\left.\right|^{6} \mid$ ChannelList $\left.\left.\right|^{3}\right)$.

Similarly, the number of generated constraints for both the multicast tree construction and the channel assignment subproblems of the Sequential method are listed in Tables 8 and 9, respectively. In short, the time complexities of the multicast tree construction and the channel assignment sub-problems are

$$
O\left(\mid \text { Nodes }\left.\right|^{6} \mid \text { Radios }\left.\right|^{6}\right) \text { and } O\left(\mid \text { Nodes }\left.\right|^{6} \log \left(\text { Nodes }^{2}\right) \mid \text { Channel }^{2}\right.
$$

List $\left.\right|^{3}$ ), respectively. Therefore, it is obvious that the time complexity of the layered design is less than that of the crosslayer design.

\section{Conclusion}

In this paper, for the first time, a comprehensive mathematical optimisation framework for the joint problem of multicast routing and channel assignment in MCMR WMNs has been proposed. Unlike the prior sub-optimal methods, which are substantially based on heuristic- and meta-heuristic-based initiatives, we have come up with a rigorous formulation to achieve the optimal solution. In particular, we have proposed two
Table 8 The number of generated constraints for the multicast tree construction sub-problem

\begin{tabular}{ll}
\hline $\begin{array}{l}\text { Constraint in multicast tree } \\
\text { construction sub-problem }\end{array}$ & $\begin{array}{l}\text { Number of constraints during } \\
\text { implementation }\end{array}$ \\
\hline$(26)$ & $\mid$ Nodes $|-|$ MulticastTarget $\mid-1$ \\
$(27)$ & $\mid$ Nodes $|-|$ MulticastTarget $\mid-1$ \\
$(28)$ & $\mid$ Nodes $\left.\right|^{2} \times \mid$ Radios $\left.\right|^{2}$ \\
$(29)$ & 1 \\
$(30)$ & 1 \\
$(31)$ & 1 \\
$(32)$ & $\mid$ MulticastTarget $\mid$ \\
$(33)$ & $\mid$ MulticastTarget $\mid$ \\
$(34)$ & $\mid$ Nodes $\left.\right|^{2} \mid$ Radios $\left.\right|^{2}$ \\
$(35)$ & $\mid$ Nodes $\left.\right|^{2} \mid$ Radios $\left.\right|^{2}$ \\
$(36)$ & $\mid$ Nodes $|-|$ MulticastTarget $\mid-1$ \\
$(37)$ & $\mid$ Nodes $|-|$ MulticastTarget $\mid-1$ \\
\hline
\end{tabular}

Table 9 The number of generated constraints for the channel assignment sub-problem

\begin{tabular}{ll}
\hline $\begin{array}{l}\text { Constraint in channel } \\
\text { assignment sub-problem }\end{array}$ & $\begin{array}{l}\text { Number of constraints during } \\
\text { implementation }\end{array}$ \\
\hline$(42)$ & $\mid$ Nodes $\left.\right|^{2} \times \mid$ ChannelList $\mid$ \\
$(43)$ & $\mid$ Nodes $\left.\right|^{2} \times \mid$ ChannelList $\mid$ \\
$(44)$ & $\mid$ Nodes $\left.\right|^{2} \times \mid$ ChannelList $\mid$ \\
\hline
\end{tabular}

BIP-based methods to achieve the optimal multicast solution. The first design solves the two underlying sub-problems (i.e., channel assignment and tree construction) conjointly and hence, yields an optimal configuration. Nevertheless, the cross-layer approach is relatively demanding in terms of computational complexity and hence, we proposed a second approach in which the two sub-problems are solved sequentially instead. As evidenced by the simulation results, however, the resultant interference is marginally worse than that of our cross-layer formulation, but its mathematical foundation still has remarkable superiority over comparable designs from prior art. The time complexity and memory demands of the two proposed designs have been investigated as well. The analytical results demonstrate that the crosslayer method needs more memory requirements and also that its time complexity is more than that of the Sequential design.

We have also briefly surveyed the existing proposals for multicast routing in MCMR WMNs, highlighting their advantages and disadvantages. Furthermore, the correctness of our designs has been demonstrated in terms of both connectivity and loopless-ness. Evaluation results derived from our simulations reveal that the proposed methods outperform the methods based on LCA, MCM, GA, SA, TS in terms of interference. Among the proposed methods, the cross-layer method gives the optimal solution for the overall problem at the price of higher complexity. The interference associated with the layered approach is only slightly higher than that of the cross-layer design, but significant compared to those of the methods based on LCA, MCM, GA, SA and TS. 


\section{References}

Akyildiz, I.F. and Wang, X. (2008) 'Cross-layer design in wireless mesh networks', IEEE Transactions on Vehicular Technology, Vol. 57, No. 2, pp.1061-1076.

Alicherry, M., Bhatia, R. and Li, L.E. (2005) ‘Joint channel assignment and routing for throughput optimization in multi-radio wireless mesh networks', 11th ACM International Conference on Mobile Computing and Networking, Cologne, Germany.

Bahl, P., Adya, A., Padhye, J. and Wolman, A. (2004) 'Reconsidering wireless systems with multiple radios', Computer Communication Review, Vol. 34, No. 5, pp.39-46.

Bazaraa, M.S., Jarvis, J.J. and Sherali, H.D. (2009) Linear Programming and Network Flows, 4th ed., John Wiley and Sons, ISBN:0470462728, 9780470462720.

Cao, J., et al. (2009) 'A novel dual-key management protocol based on a hierarchical multicast infrastructure in mobile internet', International Journal of Ad Hoc and Ubiquitous Computing, Vol. 4, Nos. 3-4, pp.183-190.

Cheng, H. and Yang, S. (2008a) 'A genetic-inspired joint multicast routing and channel assignment algorithm in wireless mesh networks', Proceedings of the Eighth Annual Workshop on Computational Intelligence, pp.159-164.

Cheng, H. and Yang, S. (2008b) 'Joint multicast routing and channel assignment in multiradio multichannel wireless mesh networks using simulated annealing', Lecture Notes in Computer Science 5361, Simulated Evolution and Learning, pp.370-380.

Cheng, H. and Yang, S.X. (2011) 'Joint qos multicast routing and channel assignment in multiradio multichannel wireless mesh networks using intelligent computational methods', Applied Soft Computing, Vol. 11, No. 2, pp.1953-1964.

Crichigno, J., Wu, M.Y. and Shu, W. (2008) 'Protocols and architectures for channel assignment in wireless mesh networks', Ad Hoc Networks, Vol. 6, No. 7, pp.1051-1077.

Das, A.K., et al. (2005) 'Optimization models for fixed channel assignment in wireless mesh networks with multiple radios', 2nd IEEE Communications Society Conference on Sensor and Ad Hoc Communications and Networks, Santa Clara, California - USA.

Ding, Y., et al. (2008) 'Channel assignment with partially overlapping channels in wireless mesh networks', 4th Annual International Conference on Wireless Internet, Brussels, Belgium.

Gopinathan, A., Li, Z. and Williamson, C. (2009) 'Optimal multicast in multi-channel multi-radio wireless networks', 16th IEEE/ ACM International Symposium on Modeling 'Analysis' and Simulation of Computer and Telecommunication Systems, Imperial College, London, UK.

Gupta, P. and Kumar, P.R. (2000) 'The capacity of wireless networks', IEEE Transactions on Information Theory, Vol. 46, No. 2, pp.388-404.

Hossain, E. and Leung, K.K. (2007) Wireless Mesh Networks: Architectures and Protocols, (edited volume), ISBN: 978-0387-68839-8, Springer.

Ilog Inc. (2011) Incline Village $n v$, http://www-01.Ibm.Com/ software/integration/optimization/cplex/

Jeong, H.J., Joung, U. and Kim, D. (2007) 'Overlay multicasting for small group application in manets', International Journal of Ad Hoc and Ubiquitous Computing, Vol. 2, No. 4, pp.215-224.
Karimi, O.B., Liu, J. and Li, Z. (2010) 'Multicast in multi-channel wireless mesh networks', Lecture Notes in Computer Science.

Keegan, B., Kowalik, K. and Davis, M. (2008) 'Optimisation of multicast routing in wireless mesh networks', Information Technology and Telecommunications, October, Galway Mayo Institute of Technology.

Kodialam, M. and Nandagopal, T. (2005) 'Characterizing the capacity region in multi-radio multi-channel wireless mesh networks', 11th ACM International Conference on Mobile Computing and Networking, pp.73-87.

Lim, S-H., Kim, C., Ko, Y-B. and Vaidya, N.H. (2009) 'Efficient multicasting for multi-channel multi-interface wireless mesh networks', IEEE Military Communications Conference, Boston, MA, pp.1-7.

Ma, L., Zhu, G. and Yin, B. (2008) 'Optimization models of multichannel assignment in multi-radio wireless mesh networks', Fourth International Conference on Wireless Communications, Networking and Mobile Computing, Dalian, pp.1-5.

Marina, M.K., Das, S.R. and Subramanian, A.P. (2010) 'A topology control approach for utilizing multiple channels in multi-radio wireless mesh networks', Computer Networks, Vol. 54, No. 2, pp.241-256.

Martinez, J. and Bafalluy, J.M. (2010) 'A survey on routing protocols that really exploit wireless mesh network features', Journal of Communication, Vol. 5, No. 3, pp.211-231.

Mohamed, A. and Alnuweiri, H. (2009) 'Wireless multicast crosslayer framework for rate allocation: Protocol design, international journal of internet protocol technology', International Journal of Internet Protocol Technology, Vol. 4, No. 1, pp.44-53.

Nguyen, H.L. and Nguyen, U.T. (2008) 'Minimum interference channel assignment for multicast in multi-radio wireless mesh networks', IEEE International Wireless Communications and Mobile Computing, Crete Island, pp.626-631.

Nguyen, H.L. and Nguyen, U.T. (2009a) 'Channel assignment for multicast in multi-channel multi-radio wireless mesh networks', Wireless Communications \& Mobile Computing, Vol. 9, No. 4, pp.557-571.

Nguyen, H.L. and Nguyen, U.T. (2009b) 'Bandwidth efficient multicast routing in multi-channel multi-radio wireless mesh networks', International Conference on Ultra Modern Telecommunications \& Workshops, St. Petersburg, pp.1-8.

Nguyen, U.T. (2008) 'On multicast routing in wireless mesh networks', Computer Communications, Vol. 31, No. 7, pp.1385-1399.

Nguyen, U.T. and $\mathrm{Xu}$, J. (2007) 'Multicast routing in wireless mesh networks: Minimum cost trees or shortest path trees?', Communications Magazine, IEEE, Vol. 45, No. 11, pp.72-77.

Palanisamy, V. and Annadurai, P. (2011) 'Trust-based clustering for multicast key distribution scheme in ad hoc network', International Journal of Internet Protocol Technology, Vol. 6, Nos. 1-2, pp.46-64.

Peng, M., Wang, Y. and Wang, W. (2007) 'Cross-layer design for tree-type routing and level-based centralised scheduling in ieee 802.16 based wireless mesh networks', Iet Communications, Vol. 1, No. 5, pp.999-1006.

Rabara, S.A. and Vijayalekshmi, S. (2011) 'Interface aware distributed multicast packet replication in ad hoc network using multicast monitoring mobile agent', International Journal of Internet Protocol Technology, Vol. 6, Nos. 1-2, pp.18-26. 
Rad, A.H.M. and Wong, V.W.S. (2006) 'Joint optimal channel assignment and congestion control for multi-channel wireless mesh networks', IEEE International Conference on Communications, Istanbul, Vol. 5, pp.1984-1989.

Ramachandran, K.N., et al. (2006) 'Interference-aware channel assignment in multi-radio wireless mesh networks', IEEE INFOCOM, Barcelona, Spains, pp.1-12.

Raniwala, A., Gopalan, K. and Chiueh, T. (2004) 'Centralized channel assignment and routing algorithms for multi-channel wireless mesh networks', ACM SIGMOBILE Mobile Computing and Communications Review, Vol. 8, No. 2, pp.50-65.

Roy, S., et al. (2008) 'High-throughput multicast routing metrics in Wireless mesh networks', Ad Hoc Networks, Vol. 6, No. 6, pp.878-899.

Ruiz, P.M. and Gomez-skarmeta, A.F. (2005) 'Approximating optimal multicast trees in wireless multi-hop networks', IEEE ISCC, pp.686-691.

Ruiz, P.M., et al. (2006) 'Efficient multicast routing in wireless mesh networks connected to internet', Proceedings of the First International Conference on Integrated Internet Ad Hoc and Sensor Networks, Nice, France.

Shittu, W., et al. (2008) 'A proposed qos multicast routing framework for next-generation wireless mesh network', IJCSNS, Vol. 8, No. 9, p.280.

Skalli, H., et al. (2007) 'Channel assignment strategies for multiradio wireless mesh networks: Issues and solutions', IEEE Communications Magazine, Vol. 45, No. 11, pp.86-93.

Subramanian, A.P., et al. (2008) 'Minimum interference channel assignment in multiradio wireless mesh networks', IEEE Transactions on Mobile Computing, pp.1459-1473.

Taha, H.A. (1975) Integer Programming, Theory, Applications, and Computations, Academic Press, New York, San Francisco, London.

Tang, J., Xue, G. and Zhang, W. (2005) 'Interference-aware topology control and QoS routing in multi-channel wireless mesh networks', ACM MobiHoc, ISBN:1-59593-004-3, pp.68-77.

Tasaki, F.H.T., Sengoku, M. and Shinoda, S. (2004) 'A new channel assignment strategy towards the wireless mesh networks', APCC'04, pp.71-75.

Vandenberghe, S.B.a.L. (2004) Convex Optimization, Cambridge University Press, USA.

Wang, X.D., Akyildiz, I.F. and Wang, W.L. (2005) 'Wireless mesh networks: a survey', International Journal of Computer and Telecommunications Networking, Vol. 47, No. 4, pp.445-487.

Yang, W.L. and Wang, L.J. (2009) 'The investigation of delayconstrained multicasting with minimum-energy consumption in static ad hoc wireless networks', International Journal of $A d$ Hoc and Ubiquitous Computing, Vol. 4, Nos. 3-4, pp.237-250.

Ye, Q. and Cheng, L. (2007) 'A lightweight approach to mobile multicasting in wireless sensor networks', International Journal of Ad Hoc and Ubiquitous Computing, Vol. 2, Nos. $1-2, \mathrm{pp} .36-45$.

Yin, Z., Li, Z. and Al, E. (2007) 'A novel channel assignment algorithm for multicast in multi-radio wireless mesh networks', ISCC, Aveiro, pp.283-288.

Yuan, J., et al. (2006) 'A cross-layer optimization framework for multihop multicast in wireless mesh networks', IEEE Journal on Selected Areas in Communications, Vol. 24, No. 11, pp.2092-2103.

Zeng, G., et al. (2007) 'Multicast algorithms for multi-channel wireless mesh networks', IEEE International Conference on Network Protocols, Beijing, pp.1-10.

Zeng, G., Wang, B., Ding, Y., Xiao, L. and Mutka, M.W. (2010) 'Efficient multicast algorithms for multichannel wireless mesh networks', IEEE Transactions on Parallel and Distributed Systems, Vol. 21, No. 1, pp.86-99.

Zhao, X., et al. (2006) 'Protecting multicast sessions in wireless mesh networks', Proceedings of 31st IEEE Conference on Local Computer Networks, pp.467-474. 